\title{
Intrinsic Neuronal Activity during Migration Controls the Recruitment of Specific Interneuron Subtypes in the Postnatal Mouse Olfactory Bulb
}

\author{
Stéphane Bugeon, Clara Haubold, Alexandre Ryzynski, Harold Cremer,* and Jean-Claude Platel* \\ Aix-Marseille University, Centre National de la Recherche Scientifique, Institut de Biologie du Développement de Marseille, Unité Mixte de \\ Recherche 7288, 13009 Marseille, France
}

\begin{abstract}
Neuronal activity has been identified as a key regulator of neuronal network development, but the impact of activity on migration and terminal positioning of interneuron subtypes is poorly understood. The absence of early subpopulation markers and the presence of intermingled migratory and postmigratory neurons make the developing cerebral cortex a difficult model to answer these questions. Postnatal neurogenesis in the subventricular zone (SVZ) offers a more accessible and compartmentalized model. Neural stem cells regionalized along the border of the lateral ventricle produce two main subtypes of neural progenitors, granule cells and periglomerular neurons that migrate tangentially in the rostral migratory stream (RMS) before migrating radially in the olfactory bulb (OB) layers. Here, we used targeted postnatal electroporation to compare the migration of these two populations in male and female mice. We do not observe any obvious differences regarding the mode of tangential or radial migration between these two subtypes. However, we find a striking increase of intrinsic calcium activity in granule cell precursors (GC-Ps) when they switch from tangential to radial migration. By decreasing neuronal excitability in GC-Ps, we find that neuronal activity has little effect on migration but is required for normal positioning and survival of GC-Ps in the OB layers. Strikingly, decreasing activity of periglomerular neuron precursors (PGN-Ps) did not impact their positioning or survival. Altogether these findings suggest that neuronal excitability plays a subtype specific role during the late stage of migration of postnatally born $\mathrm{OB}$ interneurons.
\end{abstract}

Key words: postnatal neurogenesis; migration; SVZ; olfactory bulb; neuronal activity

Significance Statement

While neuronal activity is a critical factor regulating different aspects of neurogenesis, it has been challenging to study its role during the migration of different neuronal subpopulations. Here, we use postnatal targeted electroporation to label and manipulate the two main olfactory bulb $(\mathrm{OB})$ interneuron subpopulations during their migration: granule cell and periglomerular neuron precursors (PGN-Ps). We find a very striking increase of calcium activity only in granule cell precursors (GCPs) when they switch from tangential to radial migration. Interestingly, blocking activity in GC-Ps affected mainly their positioning and survival while PGN-Ps were not affected. These results suggest that neuronal activity is required specifically for the recruitment of GC-Ps in the OB layers.

Received July 22, 2020; revised Jan. 11, 2021; accepted Jan. 15, 2021.

Author contributions: S.B., H.C., and J.-C.P. designed research; S.B., C.H., A.R., and J.-C.P. performed research; H.C. and J.-C.P. contributed unpublished reagents/analytic tools; S.B., C.H., A.R., and J.-C.P. analyzed data; S.B., H.C., and J.-C.P. wrote the paper.

This work was supported by Fédération pour la Recherche sur le Cerveau (H.C.), the Agence National pour la Recherche Grant ANR-13-BSV4-0013, ANR-17-CE16-0025-02 Fondation pour la Recherche Medicale Grants ING20150532361, Equ 201903007806 (to H.C.) and FDT20160435597 (to S.B.), and the Fondation de France Grant FDF70959 (to H.C.). We thank the (remer lab, Jenelle Wallace, and Dominique Debanne for critical reading of the manuscript. We also thank the local PiCSL-FBI core facility [Institut de Biologie du Développement de Marseille (IBDM), Aix-Marseille University] supported by the French National Research Agency through the "Investments for the Future" program (France-Biolmaging, ANR-10-INBS-04) as well as the IBDM animal facilities.

The authors declare no competing financial interests.

${ }^{*}$ H.C. and J.-C.P. are co-last authors.

Correspondence should be addressed to Harold Cremer at harold.cremer@univ-amu.fr or Jean-Claude Platel at jean-claude.platel@univ-amu.fr.

https://doi.org/10.1523/JNEUROSCl.1960-20.2021

Copyright $\odot 2021$ the authors

\section{Introduction}

Neuronal networks comprise different subtypes of interconnected neurons in a precise region-specific stoichiometry and organization. For example, in the mouse neocortex $\sim 85 \%$ of all neurons are glutamatergic, whereas $15 \%$ are inhibitory GABAergic interneurons. The mechanisms that regulate the migration and recruitment of appropriate numbers of inhibitory neurons in precise brain regions remain largely unknown.

Electrical activity has been identified as a key regulator of network development. For example, activity controls numerical population matching in the cortex. Electrical input from pyramidal neurons to interneurons determines their survival or death thereby controlling the amount of interneurons to the needs of the principal neuron pool (Lodato et al., 2011; Wong et al., 2018). 
In addition, there are evidence that electrical activity impacts on neuronal migration and consequently final positioning of cortical interneurons. For example, specific subpopulations of interneurons show a shift in laminar positioning when their activity is inhibited by the expression of the Kir2.1 channel (De Marco García, 2011).

However, despite such first observations, the specific role of neuronal activity during migration is not well understood. This is mainly due to a paucity of early markers, that allow the identification of interneuron subpopulations already during their migratory phase and before reaching terminal positions. Moreover, specifically during corticogenesis, migratory and postmigratory populations are intermingled and homogeneous compartments are not identifiable. Thus, to understand the impact of activity on interneuron migration and terminal positioning, lineage tracing and developmental models with clear compartmentalization are needed.

Activity has also been shown to be a major regulation factor of interneuron integration in the olfactory system. Indeed, in the postnatal and adult rodent, new interneurons are produced throughout life (Lois and Alvarez-Buylla, 1994) and permanently added to the preexisting circuitry of the olfactory bulb (OB; Platel et al., 2019). In this system neural stem cells localized in the ventricular-subventricular zone (V-SVZ) along lateral ventricles generate neuronal precursors that migrate tangentially via the rostral migratory stream (RMS) into the core of the OB. Here, they detach from the RMS and switch from tangential to radial migration. Granule cell precursors (GC-P) migrate short distances and settle in the granule cell layer (GCL). These represent $\sim 90 \%$ of all new neurons. Periglomerular neuron precursors (PGN-Ps) traverse the entire GCL to reach the peripheral glomerular layer (GL) before their integration. Importantly, the generation of these interneuron subtypes relies on the regionalization of the stem cell compartment in the V-SVZ. For example, GCs are lineage related to stem cells localized in lateral aspects of the ventricular wall, whereas dopaminergic/GABAergic, calretinin-positive/GABAergic, and pure GABAergic PGN are predominantly derived from stem cells in the dorsal and medial walls (Merkle et al., 2007; Fernández et al., 2011). Several lines of evidence suggest that, like in the cortex, activity is a major regulator of this interneuron integration process. For example, increasing neuronal activity via olfactory training (Mouret et al., 2008) or overexpressing the bacterial sodium channel $\mathrm{NaChBac}$ (Lin et al., 2010) enhanced the number of newborn neurons integrated. By contrast, sensory deprivation via naris occlusion decreased the survival of newborn neurons in the OB (Saghatelyan et al., 2005; Yamaguchi and Mori, 2005; Platel et al., 2019).

Altogether, the highly compartmentalized environment of the OB neurogenic system provides the possibility to trace interneuron subpopulations destined for different target layers and study their specific migratory behavior and activity patterns during tangential and radial phases. Finally, because of the fact that defined stem cell pools can be efficiently manipulated, it is particularly well suited to analyze and manipulate activity patterns.

Here, we use targeted brain electroporation to label specifically the lateral and medial ventricular wall and show that this approach labels with high predominance either future GCs or PGNs. We compared their migratory behavior and neuronal activity at high resolution, combining acute forebrain slices with two-photon microscopy. Tangential and radial migration were indistinguishable between both populations. However, GC-P showed increased calcium activity already in the distal RMS, before exit and onset of radial migration. Moreover, we find that inhibition of activity in GC-P but not in PGN-P affects the transition from tangential to radial migration, their final positioning and survival. Our results indicate that activity during migration is specifically implicated in the recruitment process of GC-P before they enter the $\mathrm{OB}$ neuronal network. In constrast, the migration of PGN-P to the OB network seems largely independent on activity.

\section{Materials and Methods}

Animals

All mice were treated according to protocols approved by the French Ethical Committee (\#5223-2016042717181477 v2). Mice were group housed in regular cages under standard conditions, with up to five mice per cage on a 12/12 h light/dark cycle. CD1 (Charles River), Ail4 (Rosa tdTomato) transgenic reporter (The Jackson Laboratory, stock \#007914) and GCaMP6s (The Jackson Laboratory, stock \#028866) mice were used. All experiments were performed on males and females.

\section{Plasmids and $m R N A$ preparation}

pCAG-Kir2.1-T2A-tdTomato (https://www.addgene.org/60598/; Xue et al., 2014), pCAG-Kir2.1Mut-T2A-tdTomato (https://www.addgene.org/ 60644/). The pCAGGS-EGFP vectors was derived from pCX-MCS2 (Morin et al., 2007). All plasmids were used at a concentration of $5 \mu \mathrm{g} / \mu \mathrm{l}$ (0.1\% Fast Green). mRNAs were provided by Miltenyi Biotec (Miltenyi Biotec): CRE Recombinase mRNA (130-101-113; a kind gift from A. Bosio, Miltenyi Biotec). For electroporation, mRNAs were diluted in RNase-free PBS at a concentration of $0.5 \mu \mathrm{g} / \mu \mathrm{l}$ (same range of molarity compared with $5 \mu \mathrm{g} / \mu \mathrm{l}$ DNA plasmid).

\section{Immunohistochemistry and image analysis}

For histologic analysis, pups were deeply xylazine/ketamine anaesthetized. Perfusion was performed intracardiacally with a solution of $4 \%$ paraformaldehyde in PBS. The brain was dissected out and incubated overnight in the same fixative at $4^{\circ} \mathrm{C}$. Sections were cut at $50 \mu \mathrm{m}$ using a microtome (Microm). Floating sections were first incubated overnight at $4^{\circ} \mathrm{C}$ with the following primary antibody: cleaved-caspase 3 antibody (cell signaling technology), before incubation $2 \mathrm{~h}$ at room temperature with the corresponding fluorescent labeled secondary antibody. Before mounting, cell nuclei were stained with Hoechst 33258. Optical images were taken either using a fluorescence microscope (Axioplan2, ApoTome System, Zeiss) or a laser confocal scanning microscope (LSM510 or LSM780, Zeiss).

For quantifications of cell distribution, layers were manually drawn based on the nuclear staining. Then, the CellCounter plugin from ImageJ was used to manually count cells in each layer. All experiments and quantifications were performed blindly to experimental groups.

\section{Postnatal electroporation}

Postnatal electroporation was performed as described previously (Boutin et al., 2008; Bugeon et al., 2017). Briefly, postnatal day (P)0P1 pups were anesthetized by hypothermia. A glass micropipette was inserted into the lateral ventricle, and $2 \mu \mathrm{l}$ of plasmid or RNA solution was injected by expiratory pressure using an aspirator tube assembly (Drummond). Successfully injected animals were subjected to five $95 \mathrm{~V}$ electrical pulses $(50 \mathrm{~ms}$, separated by $950-\mathrm{ms}$ intervals) using the CUY21 edit device (Nepagene) and $10 \mathrm{~mm}$ tweezer electrodes (CUY650P10, Nepagene) coated with conductive gel (Control Graphique Medical). Electrodes were oriented according to the desired targeting: for an electroporation of the left hemisphere, the cathode was placed on the left side and anode on the right side for lateral electroporation; for medial electroporation cathode on the right side and anode on the left side. Because of the lower number of cells generated from the medial V-SVZ, electroporations were preferentially performed at $\mathrm{P} 0$, when it is the most efficient. Electroporated animals were then reanimated in a $37^{\circ} \mathrm{C}$ incubator before returning to the mother. 
Acute brain slices

P5-P10 animals were ketamine/xylazine anesthetized, and perfused intracardiacally with cold $\left(4^{\circ} \mathrm{C}\right)$, oxygenated $\left(95 \% \mathrm{O}_{2} / 5 \% \mathrm{CO}_{2}\right)$ dissection solution ( $250 \mathrm{~mm}$ sucrose, $3 \mathrm{~mm} \mathrm{KCl}, 1.25 \mathrm{~mm} \mathrm{NaH}_{2} \mathrm{PO}_{4}, 3 \mathrm{~mm}$ $\mathrm{MgSO}_{4}, 10 \mathrm{~mm}$ D-glucose, $26 \mathrm{~mm} \mathrm{NaHCO}$, and $0.5 \mathrm{~mm} \mathrm{CaCl}_{2}$ ). The brain was then quickly removed, and glued on a vibratome platform. $300 \mu \mathrm{m}$ thick sagittal slices were taken with a vibratome (Leica VT1200S) in chilled $\left(4^{\circ} \mathrm{C}\right)$, oxygenated $\left(95 \% \mathrm{O}_{2} / 5 \% \mathrm{CO}_{2}\right)$ dissection solution. Thick sections were then placed in oxygenated DMEM, high glucose, GlutaMAX (Invitrogen), or artificial CSF (ACSF; $124 \mathrm{~mm} \mathrm{NaCl}, 3 \mathrm{~mm} \mathrm{KCl,} 1.25 \mathrm{~mm}$ $\mathrm{NaH}_{2} \mathrm{PO}_{4}, 1.3 \mathrm{~mm} \mathrm{MgSO}$, $10 \mathrm{~mm}$ D-glucose, $26 \mathrm{~mm} \mathrm{NaHCO}_{3}$, and $2 \mathrm{~mm}$ $\mathrm{CaCl}_{2}$ ) at room temperature for at least $1 \mathrm{~h}$ before imaging.

\section{Two-photon time-lapse imaging}

Acute brain slices were imaged with a two-photon Zeiss 7MP, equipped with a $20 \times$ objective (1.0 NA) and a Mai-tai laser (ONE BOX TI: SAPPHIRE LASERS, Spectra-Physics). Slices were imaged in a flowthrough warming chamber (Warner Instruments, Open Diamond Bath Imaging Chambers, RC-26G) and continuously superfused (Compact Peristaltic Pump, Harvard Apparatus; $\sim 1 \mathrm{ml} / \mathrm{min}$ ) with the appropriate recording solution bubbled with $95 \% \mathrm{O}_{2} / 5 \% \mathrm{CO}_{2}$ using a dual automatic temperature controller with an in-line heater (Warner Instruments). Slices were maintained with a slice anchor (Warner Instruments, SHD-26GH/10).

For migration, z-stacks (step size: $3 \mu \mathrm{m}$ ) were taken every 2 min for tangential migration and every $5 \mathrm{~min}$ for radial migration. All images were $1024 \times 1024$ pixels large, with an axial resolution of $0.52 \mu \mathrm{m}$. Zstack maximum projections were then realized on every time points, and registered for horizontal drifting using the StackReg plugin from ImageJ software. The number of cells being stationary at every given time point (2- or 5-min interval) was sufficient to use them as landmarks for the registration (see Movie 5).

For calcium imaging, single plane time lapse were acquired every 0.5 or $1 \mathrm{~s}$, for at least $2 \mathrm{~min}$. Only movies with low $\mathrm{Z}$ drifting were analyzed. Again, movies were registered using the StackReg plugin from ImageJ.

All acquisitions were done at least $50 \mu \mathrm{m}$ below the surface of the slice, to avoid strong slicing artefacts.

\section{Cell tracking and migration analysis}

Individual cells were manually tracked using Imaris software (Bitplane). Cell positions were then saved in individual excel files for each movie. The parameters of saltatory migration were then analyzed in $\mathrm{R}$.

Instantaneous speed at time $t$ was calculated by dividing the distance separating the positions at $\mathrm{t}-1$ and $\mathrm{t}+1$ by two time intervals. A cell was considered as moving when this speed reached $20 \mu \mathrm{m} / \mathrm{h}$ corresponding to a displacement of $1.3 \mu \mathrm{m}$ per time frame (for a 5 -min time interval), a distance sufficient to avoid noisy movements detection. From the speed vector, we could determine phases of active migration and phases of relative immobility (stationary phases). This allowed the calculation of the mean speed of nuclear translocations corresponding to the mean of all speed above $20 \mu \mathrm{m} / \mathrm{h}$ and the percentage of stationary phases which is the percentage of time points with a speed lesser than $20 \mu \mathrm{m} / \mathrm{h}$. These parameters were calculated from the first migratory phase to the last one. Displacement per hour was calculated by dividing the total distance covered during the recording divided by the time required to travel this distance. Straightness was defined as the ratio between the displacement vector length and the cumulative distance traveled. Cells with no migratory phase were not taken into account. Cells with a single migratory phase were excluded from the calculation of the percentage of stationary phases.

For quantifying the percentage of cells migrating in the superficial versus deep GCLs, we considered time lapses which were $5 \mathrm{~h}$ long. Then the GCL was divided in two equal halves (deep and superficial). A cell was considered migrating if it showed a least one migratory period during this 5 -h period.

Neurite length and swelling formation distance were measured manually with ImageJ. Mean neurite length extension was calculated over periods when neurite length was above the mean neurite length plus 2 SD. Similarly, the frequency of neurite extensions was calculated as the number of neurite extensions (mean neurite length $+2 \mathrm{SD}$ ) per hour. The threshold for the swelling distance was set at $4 \mu \mathrm{m}$, above this value it was considered as a swelling formation. From this, the frequency of swelling formation was defined as the number of swelling formation per hour. The mean duration of swelling was the mean time spent with a swelling formation.

\section{Calcium imaging analysis}

All calcium imaging experiments were performed on GCaMP6s/Ai14 mice electroporated with the RNA Cre recombinase. The expression of this calcium sensitive probe in electroporated cells allows the visualization of intracellular calcium fluctuations.

Calcium time-lapse movies were averaged over time to obtain cell contours. Averaged images were then segmented to obtain a binary mask that was treated with the "Analyze Particles" methods in ImageJ software. Automatically drawn ROIs (Region Of Interest) were then manually curated to remove lost cells, duplicates or mistakes. The mean intensity of green fluorescence for each ROI was measured over time and saved as text file. A homemade Matlab interface was used to import, filter (with running average) and normalize intensities. Calcium transients were automatically detected by the software, with a fixed threshold of $25 \% \mathrm{dF} / \mathrm{F} 0$. The end of each calcium transients was defined as more than three time points with a consecutive decrease of amplitude. Each peak was then manually curated to avoid systematic errors from the software. The mean amplitude and frequency of calcium transients from each cell were then calculated and saved.

\section{Pharmacology}

Antagonists were bath applied at the following concentrations: D-APV (Tocris Bioscience, $50 \mu \mathrm{M}$ ), NBQX (Tocris Bioscience, $10 \mu \mathrm{M}$ ), MPEP (Tocris Bioscience, $30 \mu \mathrm{M}$ ), bicuculline (Tocris Bioscience, $10 \mu \mathrm{M}$ ), strychnine (Tocris Bioscience, $20 \mu \mathrm{M}$ ), and nifedipine (Tocris Bioscience, $10 \mu \mathrm{M}$ ).

Calcium-free solution was prepared from modified ACSF (124 mM $\mathrm{NaCl}, 3 \mathrm{~mm} \mathrm{KCl}, 1.25 \mathrm{~mm} \mathrm{NaH}_{2} \mathrm{PO}_{4}, 1.3 \mathrm{~mm} \mathrm{MgSO}_{4}, 10 \mathrm{~mm} \mathrm{D}$-glucose, $26 \mathrm{~mm} \mathrm{NaHCO}_{3}$, and $1 \mathrm{~mm}$ EGTA).

\section{Experimental design and statistical analysis}

All statistical test results and data given as mean \pm SD are presented in each figure legends. For all experiments (except Fig. 3), one slice per animal was used, we therefore indicate only the number of animal used. Statistical analyses were performed using R software and R Commander Package (https://CRAN.R-project.org/package=Rcmdr). Data are presented as boxplots using the ggplot2 package, where the lower and upper hinges correspond to the first and third quartiles, and the upper (or lower) whisker extends from the hinge to the largest (or smallest) value no further than $1.5 *$ IQR from the hinge. The middle line represents the median value. Probability assignment: $p>0.05$ (not significant, ns), 0.01 $<p<0.05(*), 0.001<p<0.01(* *)$, and $p<0.001(* * *)$

For linear mixed effects model (LMEM), R package nlme (lme function) was used to fit a linear mixed model to the data with slices as a random effect and the experimental group as a fixed effect, to account for the dependance between cells analyzed in the same slices. Statistical results are given as: LMEM, $\chi^{2}$ (degrees of freedom) $=\chi^{2}$ score, $p$ value for experimental group effect [Figs. 1G, $2 D, 4 F, G$ (for frequency amd amplitude), $6 B, C, E-G]$.

For Figure $3 H, I$, the mixed model contained two fixed effects: the electroporation type (lateral vs medial) and the area of recording (RMS vs RMS-OB vs DeepGCL). Again, slices were considered as a random effect in the LMEM. Here, statistical results are given as: LMEM, $\chi^{2}$ (degrees of freedom $)=\chi^{2}$ score, $p$ value for interaction effect between electroporation type and area of recording. Post hoc $p$ values were computed using the lsmeans function ( $\mathrm{R}$ lsmeans package) and Tukey adjustment for multiple comparisons.

For Figure $3 G$ (\% active cells), a two-way anova was used with two fixed effects: the electroporation type (lateral vs medial) and the area of recording (RMS vs RMS-OB vs DeepGCL). Statistical results are given as $\mathrm{F}($ degrees of freedom $)=\mathrm{F}$ ratio, $p=p$ value for interaction effect. Tukey's HSD test was used for post hoc multiple comparisons.

Mann-Whitney-Wilcoxon tests were used to compare two groups when the number of samples was insufficient to use parametric tests: Figures $2 B, G, 4 F, G$ (\% active cells), 7C,D, 8C,D. 
A

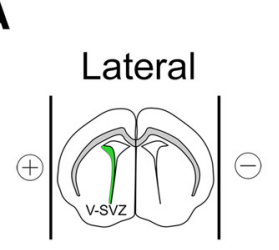

C

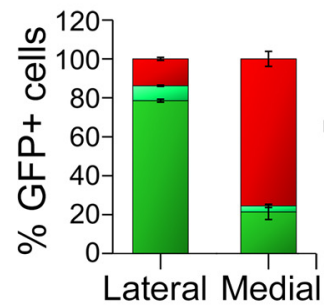

B

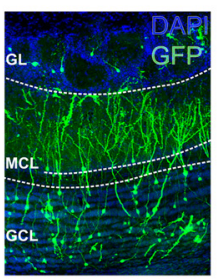

- Periglomerular neuron $\square$ Granule cells in MCL

$\square$ Granule cells

D
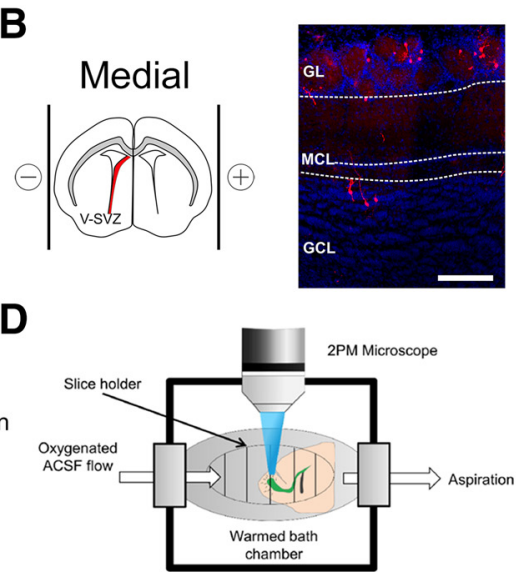

Migration recording

\section{E}

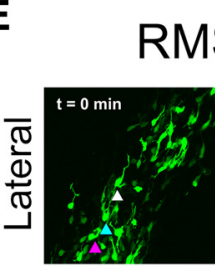

RMS Tangential migration
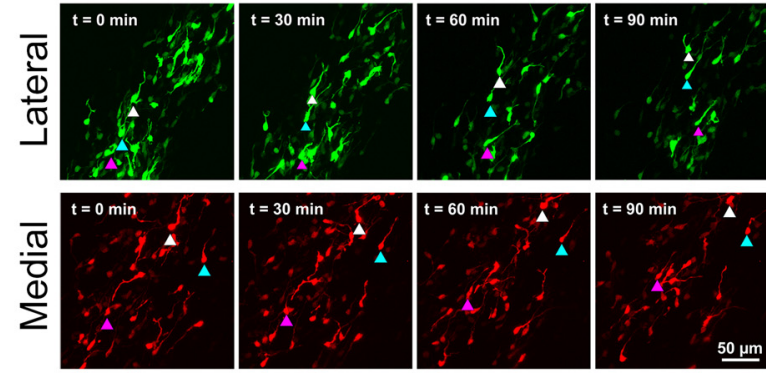

F
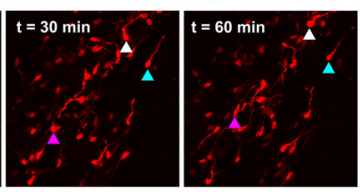

G

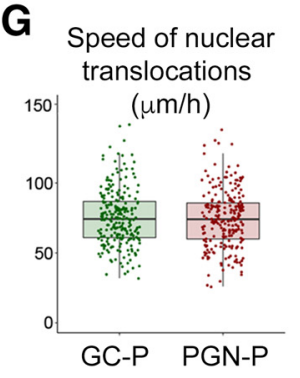

Straightness

Displacement $(\mu \mathrm{m} / \mathrm{h})$
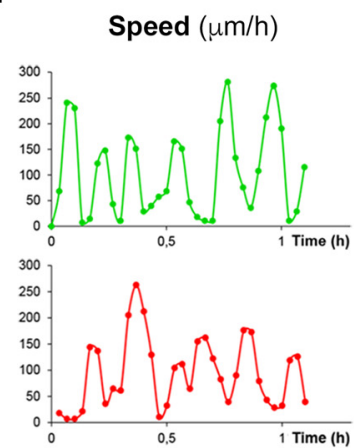

Stationary phases

(\%)
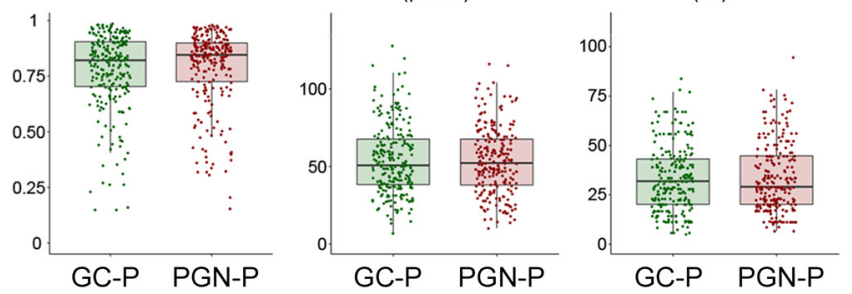

Figure 1. Tangential migration recording of postnatally generated subpopulation of $O B$ interneurons. $A$, left, Schematic of the V-SVZ from animals electroporated laterally. Right, Coronal sections of the $O B$ at 21 dpe after lateral electroporation at P1 with a GFP encoding plasmid. Most of the neurons generated by lateral stem cells are located in the GCL. $\boldsymbol{B}$, left, Schematic of the V-SVZ from animals electroporated medially. Right, Coronal sections of the OB at 21 dpe after medial electroporation at P1 with a GFP encoding plasmid. Most of the neurons generated by medial stem cells are located in the GL. Scale bar: $50 \mu \mathrm{m}$. C, Quantification of the mean percentage of GFP-positive cells in the different OB layers after lateral or medial electroporation. $\boldsymbol{D}$, Experimental setup for two-photon time lapse imaging of acute brain slices. E-G, R26-Ai14 P0 animals received lateral or medial electroporation of Cre mRNAs. The parameters of tangential migration of tdTomato-expressing neuroblasts in the RMS were then assessed on acute brain slices at 5 dpe. $\boldsymbol{E}$, Snapshots of time-lapse two-photon imaging of lateral (up) and medial cells (down) at 5 dpe. For each panel, arrowheads indicate individual cells followed over time. Time is indicated in minutes. Scale bar: $50 \mu \mathrm{m}$. $\boldsymbol{F}$, Example of instantaneous speed recorded during a 1-h movie for a lateral (up) and a medial cell (down). G. Mean speed of nuclear translocations, straightness, displacement per hour and percentage of stationary phases for RMS tangentially migrating lateral and medial cells. $n=237 / 231$ cells on three animals per condition. Speed of nuclear translocations [mean \pm SD: $75.4 \pm 20.2 \mathrm{vs} 74.2 \mathrm{SD} 20.6 \mu \mathrm{m} / \mathrm{h} ; \mathrm{LMEM}, \chi^{2}(1)=0.05$, $p=0.83$ (group effect)], straightness of cell body progression [0.78 \pm 0.17 and $0.79 \pm 0.17$; LMEM, $\chi^{2}(1)=0.09, p=0.76$ (group effect)], cell body displacement per hour [54.6 \pm 22 and $53.7 \pm 21.4 \mu \mathrm{m} / \mathrm{h} ; \mathrm{LMEM}, \chi^{2}(1)=0.01, p=0.93$ (group effect)], or percentage of time spent stationary $\left[33.2 \pm 17 \%\right.$ and $33.6 \pm 17 \%$; LMEM, $\chi^{2}(1)=0, p=0.99$ (group effect)] were similar between both populations.

Similarly, Wilcoxon rank-sum tests (paired Wilcoxon test) were used when comparing the same slices before and after addition of an antagonist: Figures $4 B-D, 5 B-F$.

\section{Results}

A new model to study interneuron migration

Previous work showed that terminal positioning of interneurons in the $\mathrm{OB}$ is dependent on the position of their respective stem cells along the walls of the lateral ventricles. Neurons born along the lateral wall tend to remain in the deep positioned GCL, whereas neurons derived from the medial wall preferentially migrate into the peripheral GL (Merkle et al., 2007; Fernández et al., 2011).

In order to quantify this correlation, we used targeted in vivo brain electroporation, which allows specific introduction of genetic material in the lateral or medial stem cell compartments (Fernández et al., 2011; Bugeon et al., 2017). Animals were electroporated at $\mathrm{P} 1$ and the $\mathrm{OB}$ was analyzed $21 \mathrm{~d}$ later $[21 \mathrm{~d}$ postelectroporation (dpe)] when newborn neurons reached the $\mathrm{OB}$ 
A

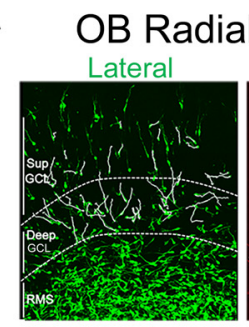

C

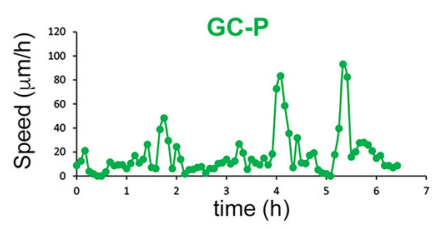

D
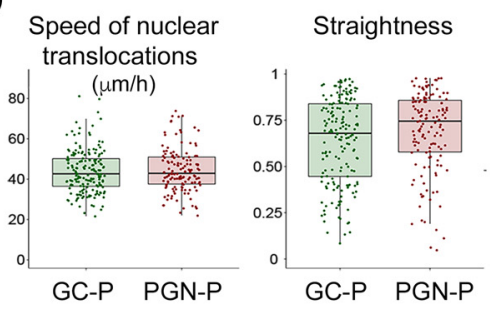

B
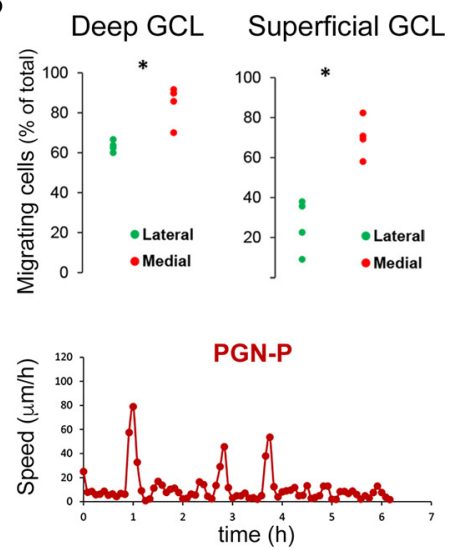

Displacement Stationary phases $(\mu \mathrm{m} / \mathrm{h})$
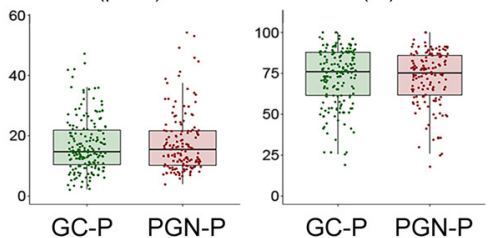

E

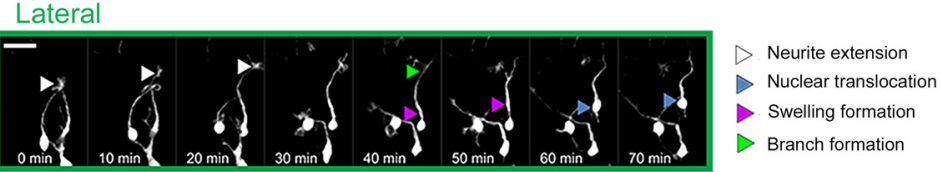

Medial

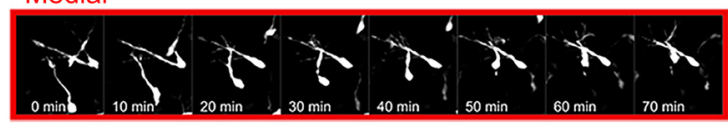

$\mathbf{F}$

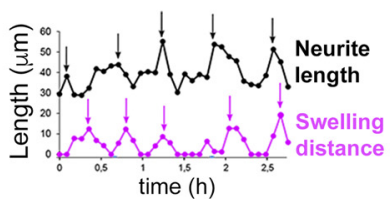

G

Mean neurite Frequency of neurite length $(\mu \mathrm{m})$ extensions $(/ \mathrm{h})$
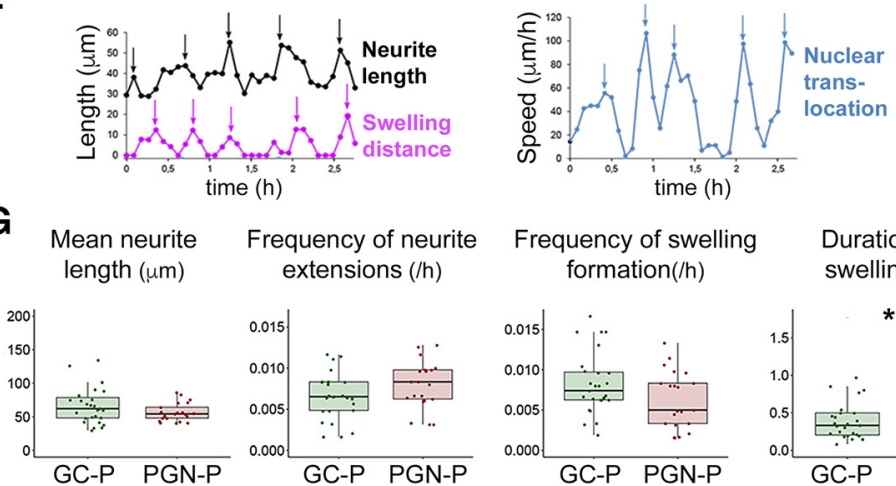

Frequency of swelling

Duration of formation $(/ \mathrm{h})$

swelling $(h)$
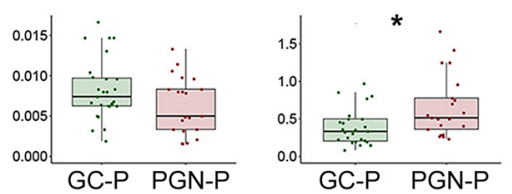

Figure 2. Characterization of radially migrating GC-P and PGN-P in the GCL. A, R26-Ai14 P0 animals received lateral or medial electroporation of Cre mRNAs. The parameters of radial migration of tdTomato-expressing neuroblasts in the GCL were then assessed on acute brain slices at 7 dpe. Example tracks (white lines) of radially migrating cells after lateral and medial electroporation. Dotted lines separate the RMS, deep GCL, and superficial GCL. Scale bar: $100 \mu \mathrm{m}$. B, Percentage of cells migrating in the deep and superficial GCL during a 5-h recording period. Cells with at least one period of active migration were considered. Each dot represents a slice, each slice comes from a different animal. $N=4$ animals. Wilcoxon rank-sum test for means: deep GCL: $W=0, p=0.03$; superficial GCL: $W=0, p=0.03$. C, Example of instantaneous speed recorded during a 6 -h movie for a lateral and a medial cell. $\boldsymbol{D}$, Mean speed of nuclear translocations, straightness, displacement per hour, and percentage of stationary phases for tangentially migrating lateral and medial cells. We found no significant differences regarding speed of nuclear translocation [44 \pm 10.7 vs $44.5 \pm 10.7 \mu \mathrm{m} / \mathrm{h} ; \mathrm{LMEM}, \chi^{2}(1)=0, p=0.99$ (group effect)], straightness [0.64 \pm 0.23 vs $0.69 \pm 0.22 ; \mathrm{LMEM}, \chi^{2}(1)=0.7, p=0.42$ (group effect)], mean displacement $\left[16.9 \pm 8.9\right.$ vs $17.8 \pm 10 \mu \mathrm{m} / \mathrm{h}$; LMEM, $\chi^{2}(1)=0.09, p=0.76$ (group effect)], or percentage of time spent in stationary phases [73.2 $\pm 17.2 \%$ vs $72.3 \pm 16.6 \%$; LMEM, $\chi^{2}(1)=0.07, p=0.8$ (group effect)] between both populations. $N=178 / 138$ cells on four animals. $E$, Snapshots of time-lapse two-photon imaging of radially migrating lateral (higher panel) and medial (lower panel) cells at high magnification. White arrowheads indicate the time of neurite extension, blue arrowheads indicate the occurrence of nuclear translocation, purple arrowheads indicate swelling formations and green arrowhead indicate a neurite branching appearance. Scale bar: $15 \mu \mathrm{m}$. $\boldsymbol{F}$, Example showing the sequence of neurite extension, swelling formation and nuclear translocation periods for one cell. Left chart, Neurite length and swelling distance are represented over time in $\mu \mathrm{m}$. Right chart, Instantaneous speed of the cells in $\mu \mathrm{m} / \mathrm{h}$. Arrows indicate the peaks of each events. $G$, Mean neurite extension length, frequency of neurite extension events, frequency and duration of swelling formations. We found no major differences in neurite length $(71 \pm 36.8$ vs $57 \pm 13.7 \mu \mathrm{m}$, Mann-Whitney-Wilcoxon test, $W=340, p=0.25)$, frequency of neurite extension $(0.007 \pm 0.003$ vs $0.008 \pm 0.003$, Mann-Whitney-Wilcoxon test, $W=203.5, p=0.1)$, frequency of swelling $(0.008 \pm 0.004$ vs $0.006 \pm 0.004$, Mann-Whitney-Wilcoxon test, $W=361, p=0.1)$ between GC-P and PGN-P. The duration of swelling $(0.44 \pm 0.36$ vs $0.66 \pm 0.42$ ) was slightly increased for $P G N-P$ (Mann-Whitney-Wilcoxon test, $W=162, p=0.012) . N=21$ cells per condition, three animals. Individual points represent cells. 

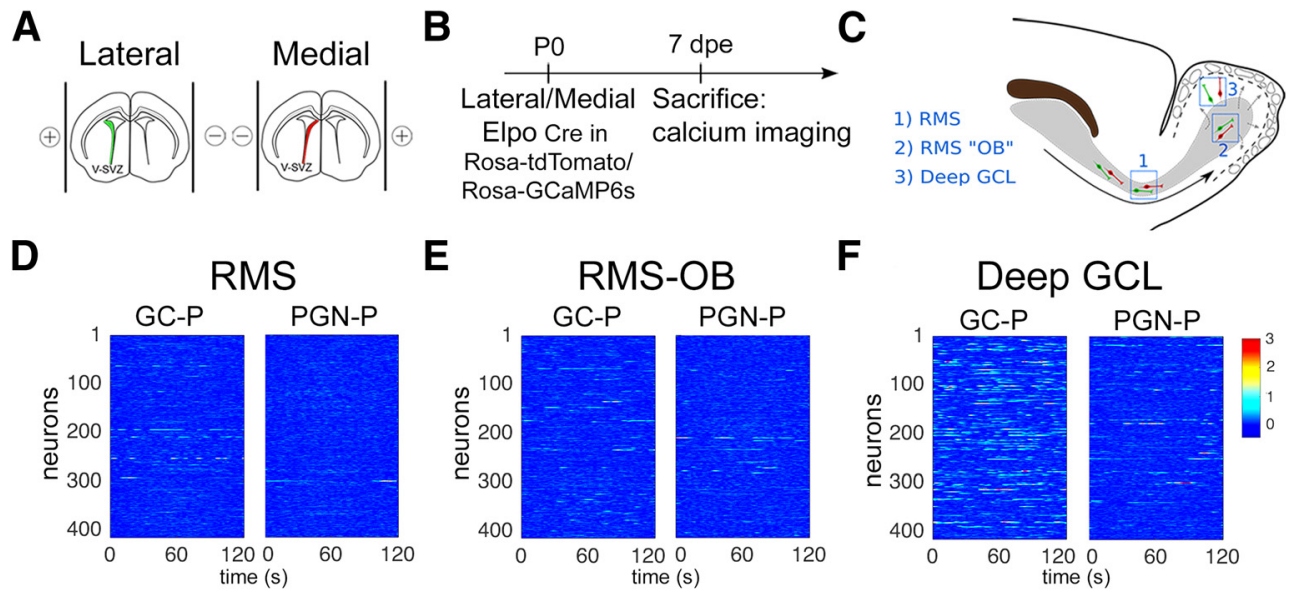

E
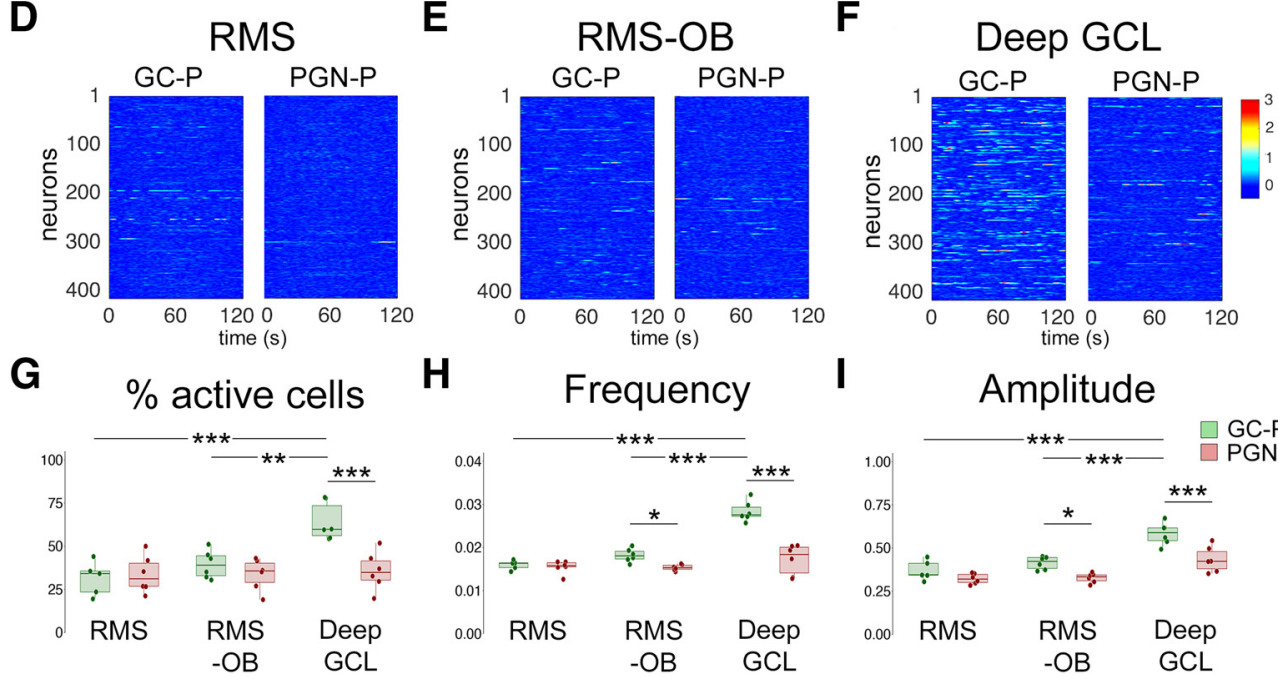

H
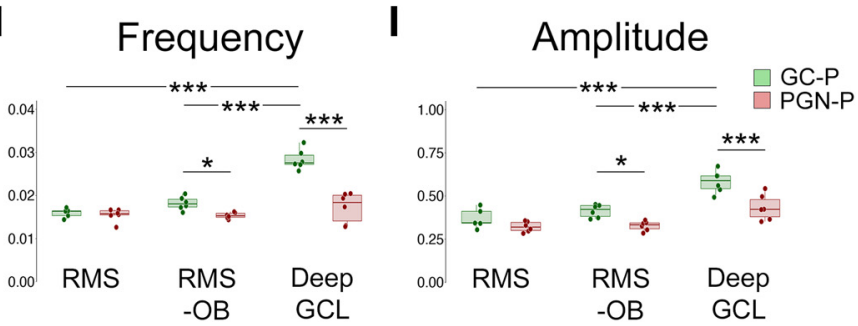

Figure 3. Spontaneous neuronal calcium activity in GC-P and PGN-P in the RMS, RMS-OB, and deep GCL. $A$, Schematic representation of an OB sagittal slice. B, Ai14/GCaMP6s P0 animals received lateral or medial electroporation of Cre mRNAs. C, Calcium activity was recorded on acute brain slices at 7 dpe. Red and green cells represent medial and lateral cells migrating in the RMS, RMS-OB, or in the deep GCL. D-F, Raster plot of calcium activity of GC-P (left) and PGN-P (right) recorded in the RMS (D), RMS-OB (E), and deep GCL (F). G-I, Percentage of active cells $(\boldsymbol{G})$, mean frequency (H), and mean amplitude $(I)$ of calcium transients for GC-P and PGN-P in the RMS, RMS-OB, and in the deep GCL. Cells were defined as active if they showed at least one detectable calcium transient. The calcium activity of GC-P greatly increased on arrival in the RMS-OB and the deep GCL, in contrast with PGN-P activity. $N=6$ slices from three animals per condition. $G$, Percentage of active cells in each slice. Two-way ANOVA, interaction effect: $\mathrm{F}(2)=7.4, p=0.0026$; Tukey's HSD test for post hoc multiple comparisons: RMS-GCp versus DeepGCL-GCp, $p=0.00012$; RMS-GCp versus RMS-0B-GCp, $p=0.0024$; DeepGCL-GCp versus DeepGCL-PGNp, $p=0.00042$. H, Mean frequency of calcium transients. $N=624 / 1269 / 730$ versus 288/537/139 (RMS/RMS-0B/Deep-GCL) cells. LMEM, $\chi^{2}(2)=35.6, p<0.0001$ (interaction effect); least-squares means and Tukey adjustment for post hoc multiple comparisons: RMS-GCp versus DeepGCL-GCp, $p<0.0001$; RMS-GCp versus RMS-OB-GCp, $p<0.0001$; RMS-0B-GCp versus RMS-OB-PGNp, $p=0.049$; DeepGCL-GCp versus DeepGCL-PGNp, $p=0.0001$. I, Mean amplitude of calcium transients. $N=624 / 1269 / 730$ versus 288/537/139 (RMS/RMS-0B/Deep-GCL) cells. LMEM, $\chi^{2}(2)=8.6, p=0.014$ (interaction effect); least-squares means and Tukey adjustment for post hoc multiple comparisons: RMS-GCp versus DeepGCL-GCp, $p<0.0001$; RMS-GCp versus RMS-0B-GCp, $p$ $<0.0001$; RMS-0B-GCp versus RMS-OB-PGNp, $p=0.028$; DeepGCL-GCp versus DeepGCL-PGNp, $p=0.0001$

and settled in their terminal positions. For clarity, we use falsecolor representations, lateral electroporation in green and medial in red, throughout the manuscript. Introduction of a GFPexpression plasmid pCAG-GFP into the lateral ventricular wall labeled with high predominance $(\sim 80 \%)$ granule cells that remained in central positions within the GCL. Only few labeled neurons were found in the mitral cell layer (MCL) and the GL (Fig. 1A,C). In contrast, electroporation of pCAG-GFP into the medial wall labeled mainly cells that traversed the GCL to settle in the GL as PGN ( $80 \%$; Fig. $1 B, C)$.

Thus, targeted electroporation of the lateral or the medial stem cell compartments labeled with high predominance the lineages that led to GCs and PGNs, respectively. We used this model to study their migratory behavior.

Migratory behavior of GC-P and PGN-P is indistinguishable First, we compared GC-P and PGN-P during their tangential migration in the RMS using two-photon time-lapse imaging on acute brain slices at $5 \mathrm{~d}$ after lateral or medial electroporation (Fig. 1D,E). To increase the number of labeled cells for precise quantitative analyses, we used CRE-mRNA electroporation into R26 tdTomato transgenic mice. This approach allows specific targeting of the ventricular stem cell compartments at higher transfection efficiency (Bugeon et al., 2017).

Individual cells were manually tracked using Imaris software (Bitplane). The instantaneous speed and other migratory parameters of the cells were then derived based on their tracked position at each frame of the time lapse. Speed profiling of cell bodies (Fig. $1 F$ ), showed typical saltatory migration as reported before (Bellion et al., 2005; Nam et al., 2007; Inada et al., 2011), alternating short stationary phases and fast nuclear translocations, for both populations (Movie 1 for lateral electroporation; Movie 2 for medial electroporation). Speed of nuclear translocations, straightness of cell body progression, cell body displacement per hour and lengths of stationary phases were indistinguishable between both populations (Fig. 1G).

We then compared radial migration of both populations on acute brain slices at $7 \mathrm{dpe}$, a time point when large numbers of cells leave the RMS-OB and initiate radial migration to invade the $\mathrm{OB}$ target layers (Fig. 2A; Movie 3 for lateral electroporation; Movie 4 for medial electroporation). Quantification in the deeper half of the GCL during $5 \mathrm{~h}$ showed that $\sim 60 \%$ of the GC-P and $80 \%$ of PGN-P had migrated during the recording period (Fig. $2 B$, left). The difference in migration between the two populations was even larger when we analyzed cells located in the superficial half of the GCL. Here, $<30 \%$ of GC precursors, but still almost $80 \%$ of PGN-P, changed position during the recording period (Fig. $2 B$, right). For the migratory fractions of both populations, radial migration was still saltatory, like in the RMS, but with lower speed of nuclear translocation as compared with tangential migration (Fig. $2 C$; tangential: $75 \mu \mathrm{m} / \mathrm{h}$; radial: $45 \mu \mathrm{m} / \mathrm{h}$; Fig. 2D). Comparison of the different migratory parameters at 
A Lateral Medial
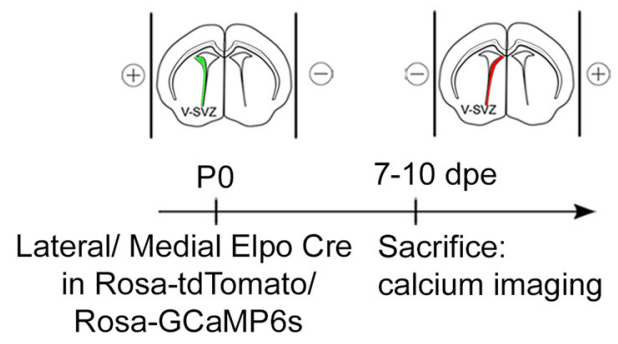

C

Lateral Elpo nifedipine
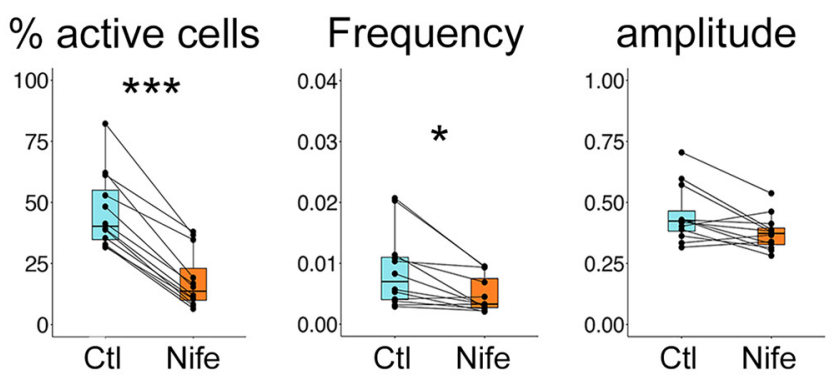

\section{E}

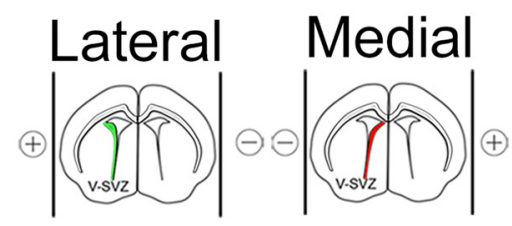

\section{Lateral Elpo: Kir2.1}

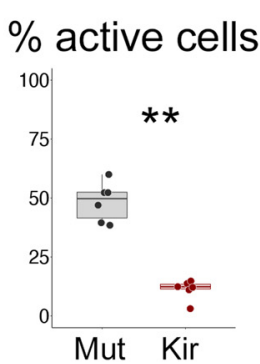

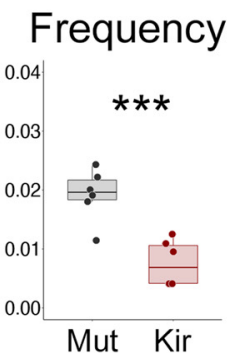

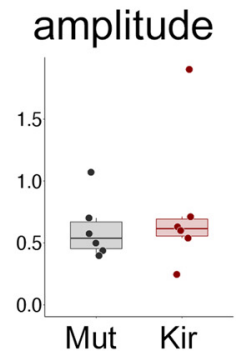

B

Lateral Elpo: 0 Calcium + EGTA

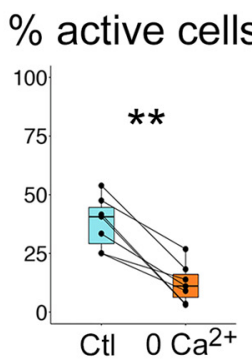

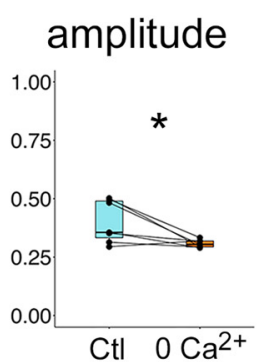

D

Medial Elpo nifedipine
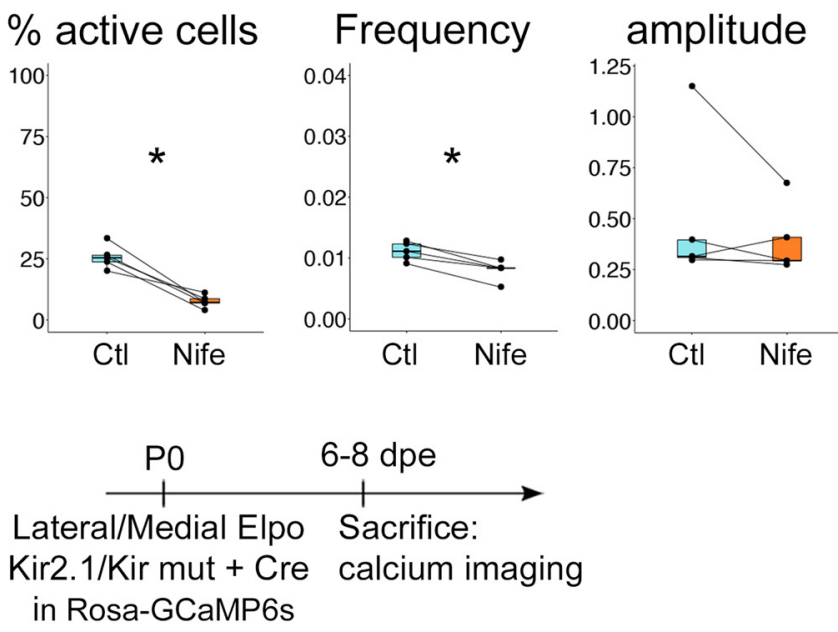

G

Medial Elpo: Kir2.1
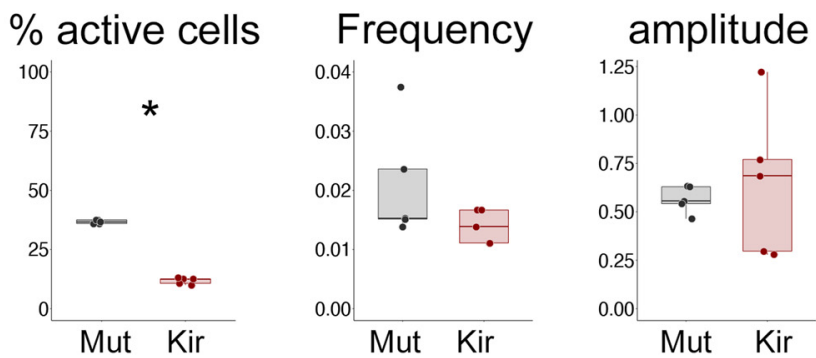

Figure 4. Spontaneous neuronal calcium activity in both GC-P and PGN-P can be partly blocked by L type-VGCC antagonist or KIR2.1 overexpression. $A$, Rosa-tdTomato/RosaGCaMP6s P0 animals were electroporated with Cre recombinase, targeting either the lateral or medial wall of the V-SVZ. 7-10 d after electroporation the animals were killed, and acute brain slices were obtained and calcium activity was recorded in the deep GCL. B, Percentage of active cells (left), mean frequency (middle), and mean amplitude (right) of calcium transients for GC-P, before and after incubation in calcium-free medium (no calcium $+2 \mathrm{~mm} \mathrm{EGTA).} \mathrm{Both} \mathrm{the} \mathrm{percentage} \mathrm{of} \mathrm{active} \mathrm{cells} \mathrm{and} \mathrm{amplitude} \mathrm{of} \mathrm{calcium} \mathrm{tran-}$ sients were significantly reduced, suggesting that the observed calcium transients require an extracellular source for calcium. $N=7$ animals. Percentage of active cells: Wilcoxon rank-sum test, $p=0.005$. Mean frequency: Wilcoxon rank-sum test, $p=0.16$. Mean amplitude: Wilcoxon rank-sum test, $p=0.038$. C, Percentage of active cells (left), mean frequency (middle), and mean amplitude (right) of calcium transients for GC-P, before and after incubation in a nifedipine (10 $\mu \mathrm{m})$ supplemented medium. Both the percentage of active cells and frequency of calcium transients were significantly reduced, suggesting that the observed calcium transients depend at least partly on L-type voltage gated calcium channels. $N=$ animals. Percentage of active cells: Wilcoxon rank-sum test, $p=0.00014$. Mean frequency: Wilcoxon rank-sum test, $p=0.04$. Mean amplitude: Wilcoxon rank-sum test, $p=0.06$. D. Percentage of active cells (left), mean frequency (middle), and mean amplitude (right) of calcium transients for PGN-P, before and after incubation in a nifedipine $(10 \mu \mathrm{m})$ supplemented medium. Both the percentage of active cells and frequency of calcium transients were significantly reduced, suggesting that the observed calcium transients depend at least partly on L-type voltage gated calcium channels. $N=5$ animals. Percentage of active cells: Wilcoxon rank-sum test, $p=0.012$. Mean frequency: Wilcoxon rank-sum test, $p=0.012$. Mean amplitude: Wilcoxon rank-sum test, $p=0.42$. $E$, Wild-type P0 animals were electroporated with either a KIR2.1 mutant form or the normal KIR2.1 channel, targeting either the lateral or medial wall of the V-SVZ; 6-8 d after electroporation, the animals were killed, and acute brain slices were obtained and imaged for calcium activity in the deep GCL. F, Percentage of active cells (left), mean frequency (middle), and mean amplitude (right) of calcium transients for GC-P, with the mutant or normal KIR2.1 channel. Both the percentage of active cells and frequency of calcium transients were significantly reduced, showing that the overexpression of the KIR2.1 channel in GC-P significantly decreases calcium activity. $N=6$ animals. Percentage of active cells: $48.3 \pm 8.2$ versus $11.2 \pm 4.1$, Mann-Whitney-Wilcoxon test, $p=0.0022, W=36$. Mean frequency: $0.019 \pm 0.004$ versus $0.0076 \pm 0.0038, \operatorname{LMEM}, \chi^{2}(1)=13, p=0.0003$ (group effect). Mean amplitude: $0.61 \pm 0.25$ versus $0.77 \pm 0.57$, LMEM, $\chi^{2}(1)=2.2, p=0.14$ (group effect). G, Percentage of active cells (left), mean frequency (middle), and mean amplitude (right) of calcium transients for PGN-P, with the 
A

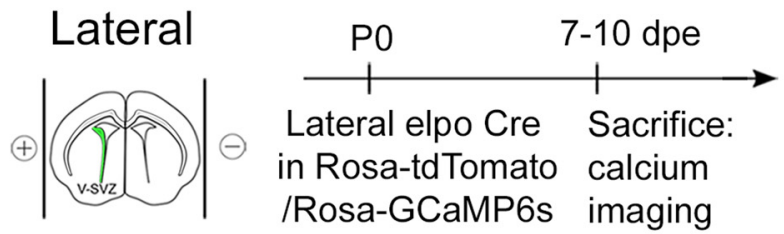

C

AMPA/KAINATE-Receptors
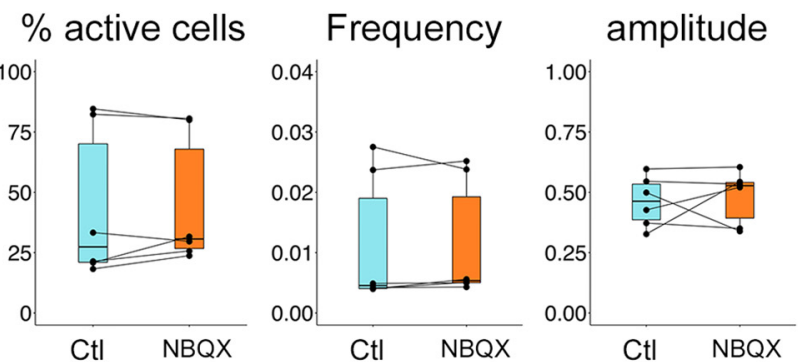

E

\section{Glycine-Receptors}

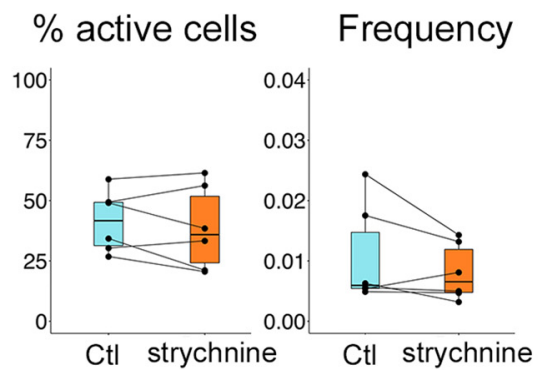

B

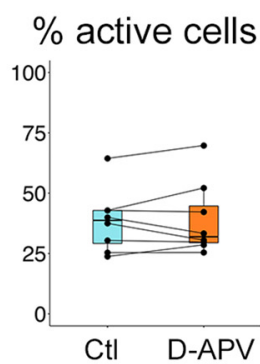

D

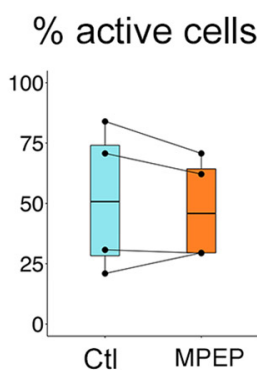

\section{mGlu-Receptors 5}

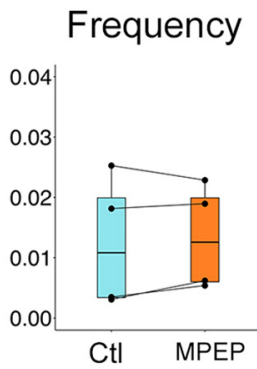

NMDA-Receptors
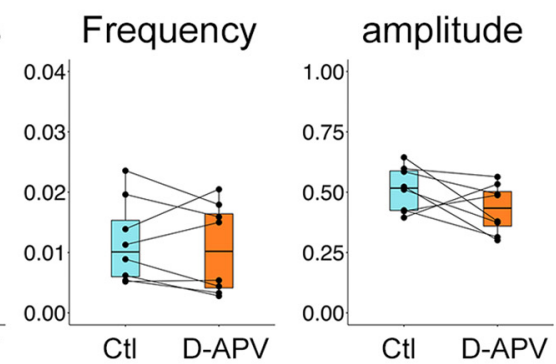

$\mathbf{F}$

$\mathrm{GABA}_{A}-$ Receptors
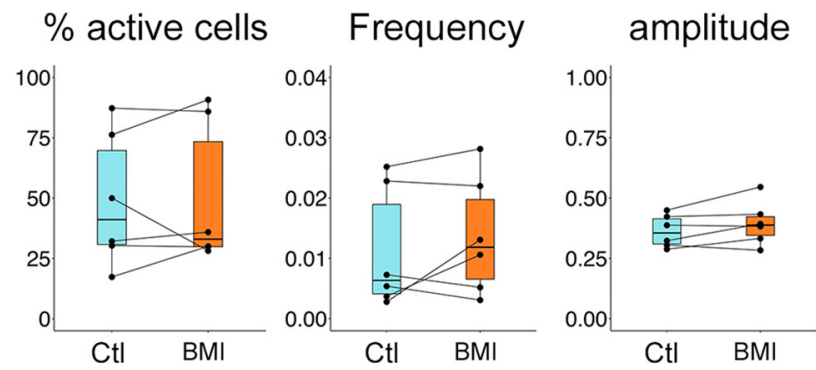

Figure 5. Spontaneous neuronal calcium activity in GC-P in the deep GCL is not affected by classical antagonists. $A$, Rosa-tdTomato/Rosa-GCaMP6s P0 animals were electroporated with a Cre recombinase, targeting the lateral wall of the V-SVZ; 7-10 d after electroporation, the animals were killed, and acute brain slices were obtained and imaged for calcium activity in the deep $G C L$. $\boldsymbol{B}-\boldsymbol{F}$, Spontaneous activity was recorded on each slice. The sections were then incubated with a given antagonist for $1 \mathrm{~h}$, then calcium imaging was performed again to compare spontaneous activity with or without antagonist. $\boldsymbol{B}$, The NMDA receptors antagonist D-APV ( $N=8$ animals; percentage of active cells: Wilcoxon rank-sum test, $p=1$; mean frequency: Wilcoxon ranksum test, $p=0.65$; mean amplitude: Wilcoxon rank-sum test, $p=0.1)$; (C) AMPA/kainate receptors antagonist NBQX ( $N=6$ animals; percentage of active cells: Wilcoxon rank-sum test, $p=0.82$; mean frequency: Wilcoxon rank-sum test, $p=0.3$; mean amplitude: Wilcoxon rank-sum test, $p=0.82) ;(\boldsymbol{D})$ metabotropic glutamate receptor 5 antagonist MPEP $(N=4$ animals; percentage of active cells: Wilcoxon rank-sum test, $p=0.69$; mean frequency: Wilcoxon rank-sum test, $p=0.69$; mean amplitude: Wilcoxon rank-sum test, $p=0.49) ;(\boldsymbol{E})$ glycine receptor antagonist strychnine ( $N=6$ animals; percentage of active cells: Wilcoxon rank-sum test, $p=0.82$; mean frequency: Wilcoxon rank-sum test, $p=0.48$; mean amplitude: Wilcoxon rank-sum test, $p=1)$; and $(\boldsymbol{F}) G_{A B A_{A}}$ receptors antagonist bicuculline $(N=7$ animals; percentage of active cells: Wilcoxon rank-sum test, $p=0.94$; mean frequency: Wilcoxon rank-sum test, $p=0.7$; mean amplitude: Wilcoxon rank-sum test, $p=0.7$ ) did not modify the percentage of active cells, frequency, or amplitude of calcium transient.

the population level showed no significant differences regarding the speed of nuclear translocation, displacement or percentage of time spent in stationary phases. Next, we compared migratory parameters in the OB of GC-P and PGN-P at the cellular level. The example presented in Figure $2 E$ provides a high-resolution view of the sequence of events during which peaks in neurite length precede swelling formation and nuclear translocation

mutant or normal KIR2.1 channel. The percentage of active cells was significantly reduced, showing that the overexpression of the KIR2.1 channel in PGN-P significantly decreases calcium activity. $N=5$ animals. Percentage of active cells: $36.7 \pm 0.75$ versus $11.7 \pm 1.3$, MannWhitney-Wilcoxon test, $p=0.0114, W=25$. Mean frequency: $0.021 \pm 0.0099$ versus $0.014 \pm 0.0027$, LMEM, $\chi^{2}(1)=1.6, p=0.21$ (group effect). Mean amplitude: $0.56 \pm 0.069$ versus $0.65 \pm 0.39$, LMEM, $\chi^{2}(1)=1.05, p=0.3$ (group effect).
(Fig. 2F; Movie 6 for lateral electroporation; Movie 7 for medial electroporation). Neurite length, frequency of neurite extension and frequency of swelling formation were similar between populations. There was a tendency toward a small but significant increase in swelling duration between GC-P and PGN-P (Fig. $2 G)$. This result may indicate a different swelling formation mechanism between these two populations.

Overall, we conclude that GC-P and PGN-P share unique modes of tangential and radial migration toward and within the OB. The significantly lower mobility of GC-P in the GC likely reflects the fact that this population is rapidly recruited to the circuitry and stop migrating. In contrast, PGN-P do not stop migration as they traverse the GCL and integrate the PGL. A clear morphokinetic predictor of neuronal fate during tangential or radial migration was not detected. 


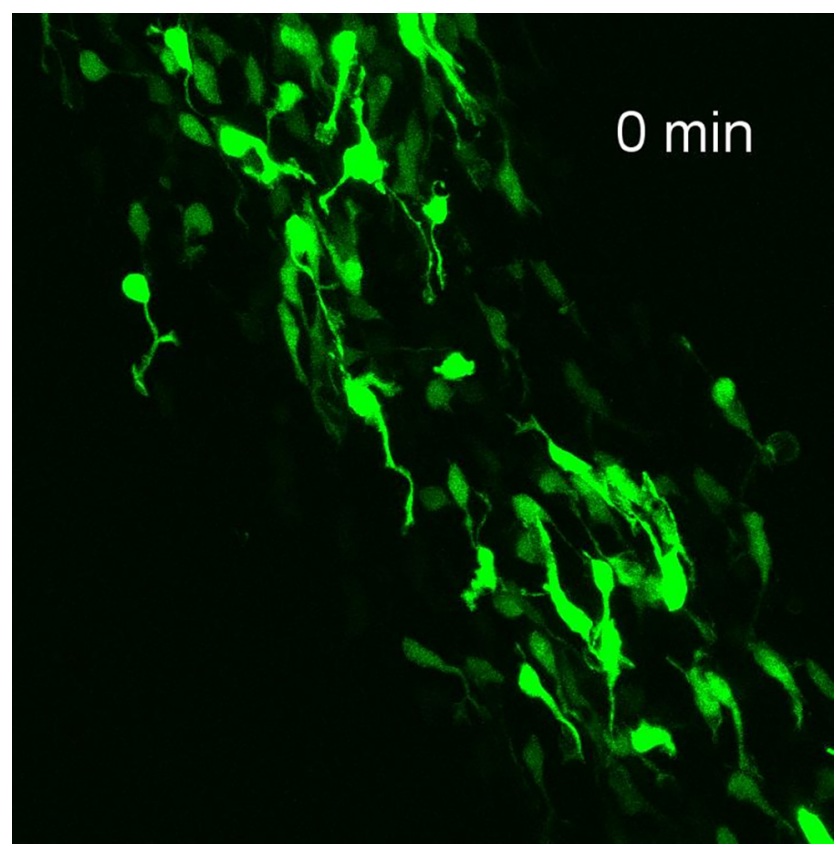

Movie 1. Example of a 70-min-long time lapse recording performed in the RMS $5 \mathrm{~d}$ after lateral electroporation. [View online]

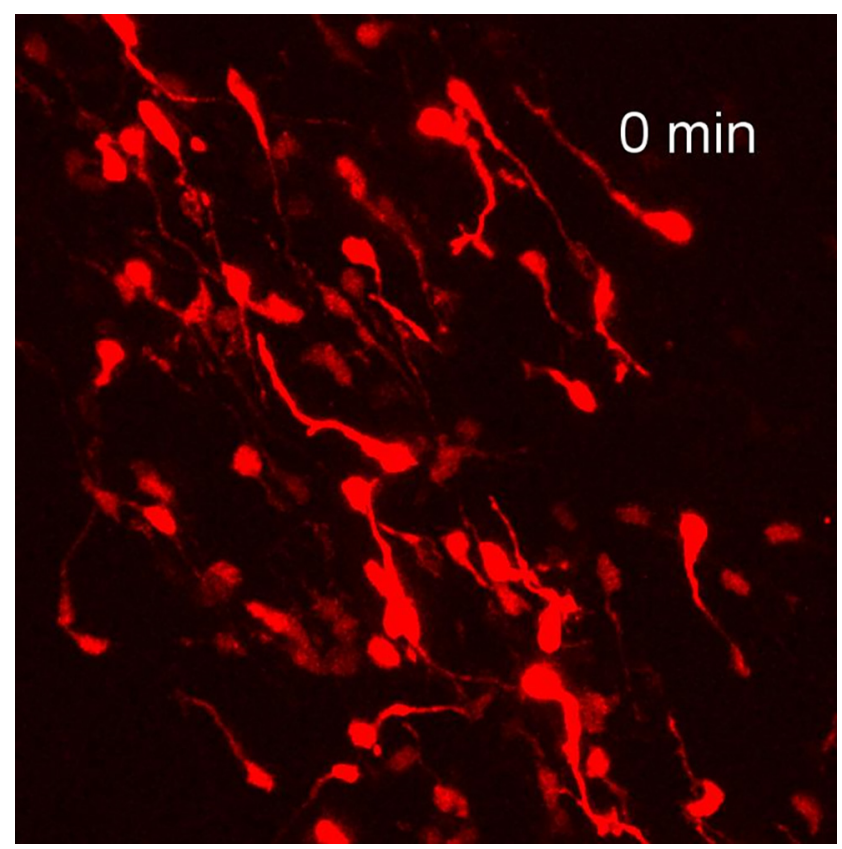

Movie 2. Example of a 70-min-long time lapse recording performed in the RMS $5 \mathrm{~d}$ after medial electroporation. [View online]

\section{Neuronal activity increases in GC-P during recruitment in the $\mathrm{OB}$}

To investigate the impact of neuronal activity on migration and recruitment of GC-P and PGN-P in the OB neurogenic system, we quantified their spontaneous calcium activity. CRE-mRNA was targeted to either the lateral or the medial stem cell compartments of R26 tdTomato/GCaMP6s mice. Acute slices were prepared and imaged at 7 dpe by two-photon microscopy (Fig. $3 A$, $B$ ). Neuronal activity in GC-P and PGN-P was observed in three defined regions (Fig. 3C): first, in the RMS, before precursors

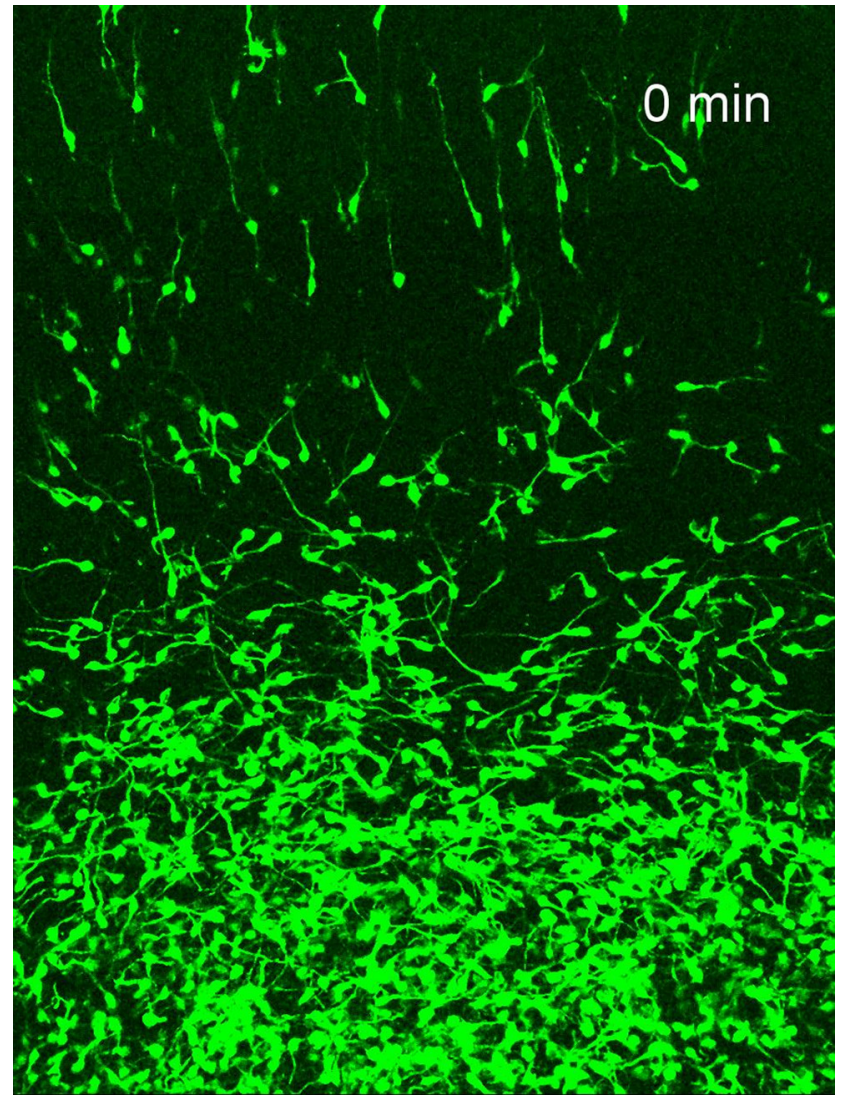

Movie 3. Example of a 160-min-long time lapse recording performed in the $O B \quad 7 \mathrm{~d}$ after lateral electroporation. [View online]

enter the core of the OB; second, in the RMS-OB before the precursors enter in the OB; third, in the deep GCL. We observed an overall increase of calcium activity (\% of active cells, frequency and amplitude, respectively Fig. 3G-I) between the RMS and the deep-GCL and between the RMS-OB and the deep-GCL for the GC-P.

In the RMS, about one third of the imaged cells were active during a 2-min recording (i.e., at least one detectable transient) with no significant differences between medial and lateral slices (Fig. 3D,G). Similarly, the amplitude and frequency of calcium transients were comparable for the two populations, with a mean of $35 \% \mathrm{dF} / \mathrm{F} 0$ at a frequency of $0.016 \mathrm{~Hz}$ (Fig. $3 \mathrm{H}, I)$. We then recorded spontaneous calcium activity in precursors located in the RMS-OB (Fig. 3E, Movie 8, Movie 9). While the percentage of active cells was similar between GC-P and PGN-P (Fig. 3G), we observed a significant increase of the frequency and amplitude of calcium transient in GC-P (Fig. 3H,I). When precursors migrated in the deep GCL (Fig. $3 F$ ), we observed an important increase of activity in GC-P compared with PGN-P. Indeed, the percentage of active cells was almost twice as large in GC-P (Fig. $3 G$ ), while there was respectively a $65 \%$ and $37 \%$ increase in amplitude and frequency of calcium transients in GC-P compared with PGN-P (Fig. 3H,I). Further statistical analysis revealed that for all parameters, there is a significant interaction between the type of electroporation (lateral vs medial) and the area where cells were recorded (RMS, RMS-OB, and deep GCL). This shows that the two subpopulations are different, and that this difference varies among layers. Indeed, while the activity of GC-Ps significantly increases in the GCL, this is not the case for PGN-Ps. 


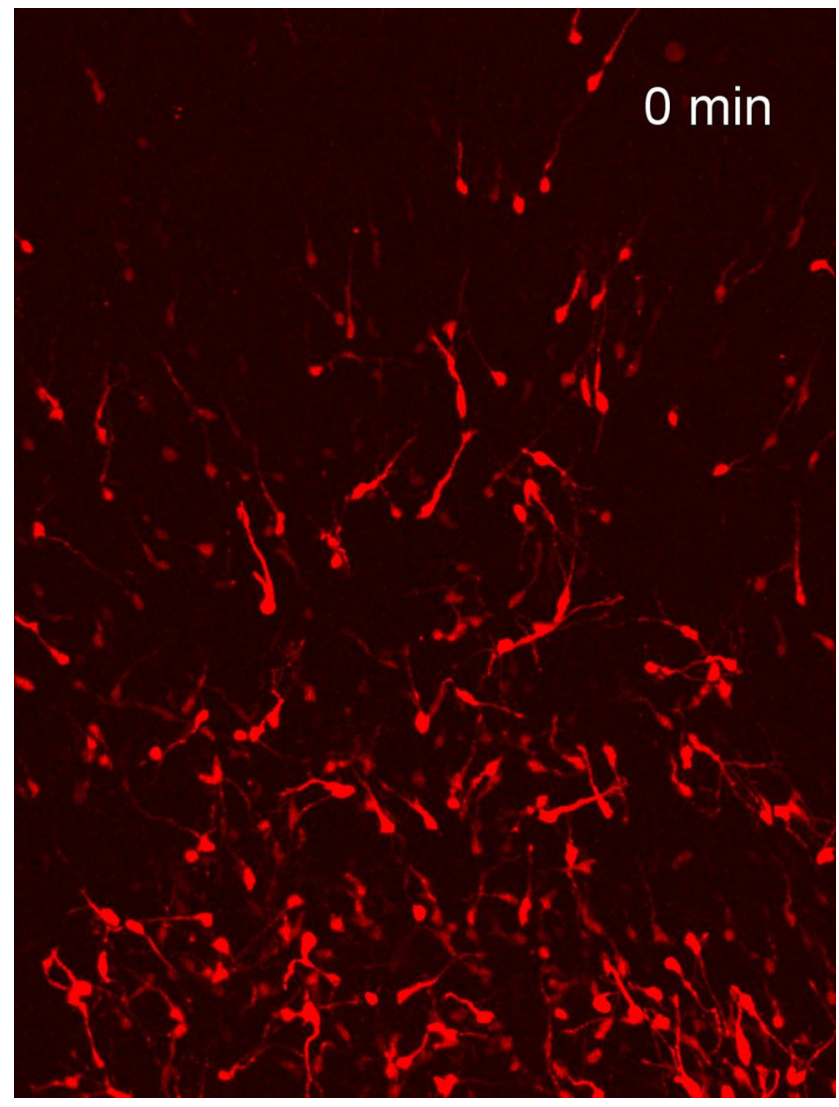

Movie 4. Example of a 160-min-long time lapse recording performed in the $O B \quad 7 d$ after medial electroporation. [View online]

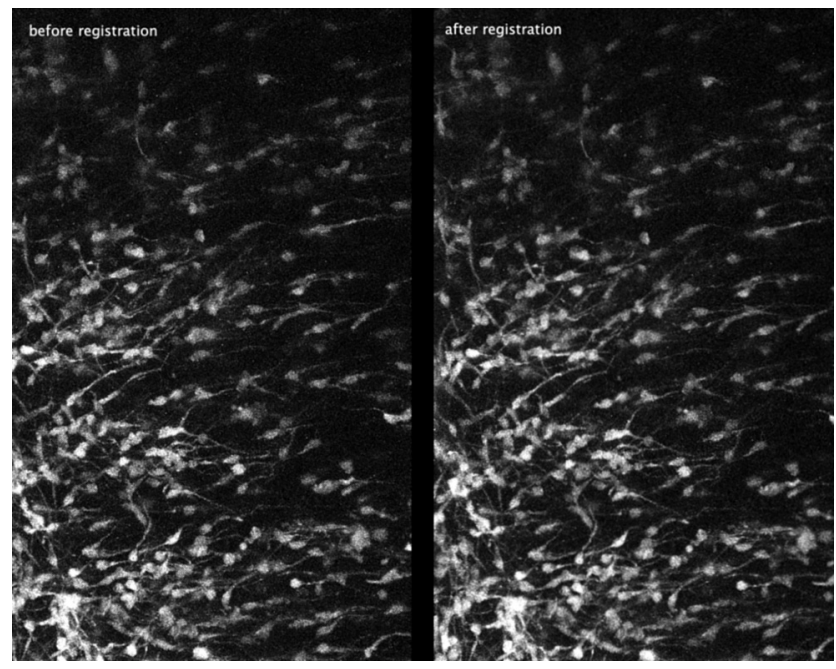

Movie 5. Example of time lapse recording before (left) and after image registration (right) to correct for horizontal drift. [View online]

We aimed at identifying the neurophysiological mechanisms underlying this increased activity of GC-P in the deep GCL (Fig. 4A). Perfusion of a calcium-free medium on $\mathrm{OB}$ slices led to a strong decrease in the percentage of active cells ( $41 \%$ vs $11 \%, p=0.004$; Fig. $4 B$ ) suggesting that the source of calcium was mainly extracellular. Moreover, blocking specifically L-type voltage gated calcium channels with nifedipine abolished most calcium activity in GC-P (Fig. 4C) indicating that a depolarization was responsible for the increase of

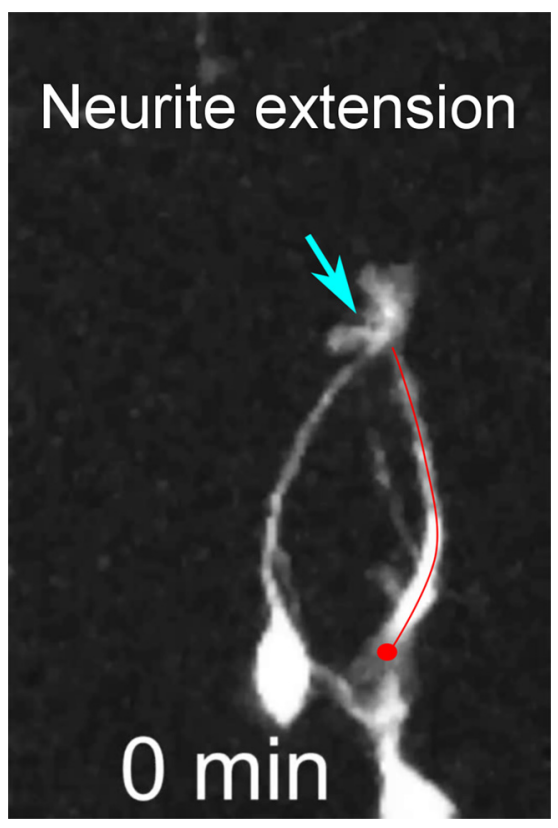

Movie 6. Example of a 110-min-long time lapse recording performed in the $O B 7 \mathrm{~d}$ after lateral electroporation showing the different phases of neuronal migration: neurite extension, branch formation, swelling formation, and nuclear translocation. [View online]

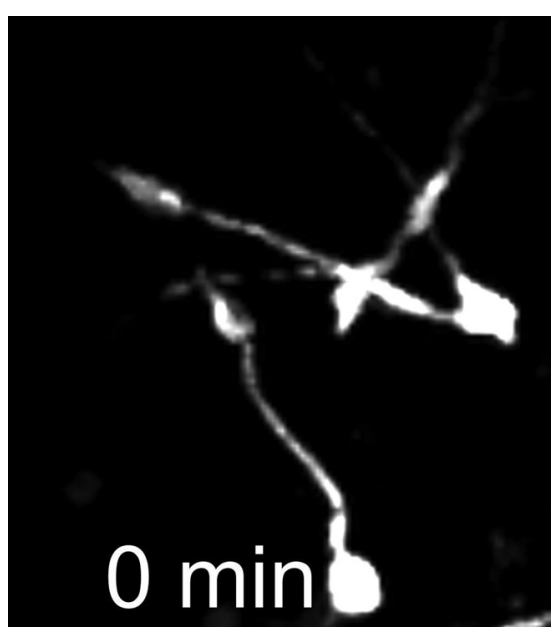

Movie 7. Example of a 110-min-long time lapse recording performed in the $O B 7 \mathrm{~d}$ after medial electroporation showing the different phases of neuronal migration: neurite extension, branch formation, swelling formation, and nuclear translocation. [View online]

activity. While the level of activity was lower in PGN-P, activity could still be inhibited by nifedipine application (Fig. $4 D)$. It was demonstrated that overexpression of the inward rectifying potassium channel Kir2.1 could highly affect neuronal activity by lowering the resting membrane potential, therefore altering neuronal excitability (De Marco García et al., 2011; Xue et al., 2014). We overexpressed Kir2.1 in GC-P and PGN-P and measured its effect on spontaneous calcium activity (Fig. 4E). We observed an important decrease of neuronal activity in GC-P (Fig. 4F). While neuronal activity was lower in PGN-P, it could still be inhibited by Kir expression (Fig. 4G).

We used a wide spectrum of pharmacological interventions to find the source of this depolarization, including the NMDA receptors antagonist $\mathrm{D}-\mathrm{APV}$, the AMPA receptors antagonist NBQX, the metabotropic glutamate receptor 5 antagonist MPEP, 


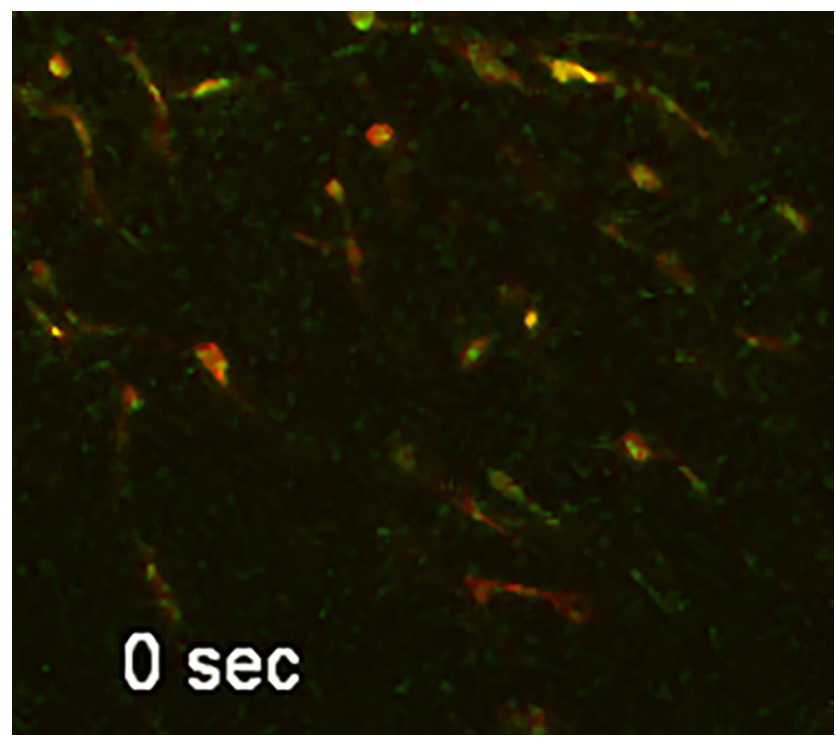

Movie 8. Example of a 2-min recording of calcium activity performed at the exit of the RMS-OB $7 \mathrm{~d}$ after lateral electroporation. GCaMP6s fluorescence is in green, tdTomato in red. [View online]

the $\mathrm{GABA}_{\mathrm{A}}$ receptors antagonist bicuculline, the glycine receptor antagonist strychnine (Fig. 5A-F). None of these antagonists significantly modified the percentage of active cells, or the amplitude or frequency of calcium transients. Taken together, these results suggest that the calcium activity increase seen in GC-P was mainly due to a depolarization, leading to the opening of voltage-dependent L-type calcium channels and an entry of extracellular calcium.

Thus, after entering the RMS in the $\mathrm{OB}$, and especially when they start exiting the RMS, GC-P, but not PGN-P, showed a large L-type calcium channel-dependent increase in neuronal activity. These results led us to hypothesize that the depolarization of GC-P during the transition from the RMS into the GCL is critical for their recruitment in the $\mathrm{OB}$.

\section{Neuronal activity is needed for recruitment and survival of GC-P}

To test whether neuronal activity controls the migration and the recruitment of precursors from the RMS to the OB layers, we interfered with the activity of arriving GC-P.

First, we investigated whether blocking neuronal activity of GC-P with nifedipine could affect their migration. We recorded neuronal migration at 7-8 dpe in presence of nifedipine in the RMS-OB and in the deep-GCL (Fig. 6A). We did not find any significant change in the speed of nuclear translocations, straightness, cell displacement and stationary phases (Fig. 6C,D).

Then we overexpressed either Kir2.1 to block neuronal activity in GC-P or Kir2.1 mutant as a control and recorded migration at 7-8 dpe (Fig. 6D). In the RMS, expression of Kir2.1 compared with Kir2.1 mutant had no effect on the speed of nuclear translocations, straightness, cell displacement and stationary phases (Fig. 6E). Next, we analyzed migration in the RMS$\mathrm{OB}$, a region where neuronal activity is increased in GC-P compared with PGN-P (Fig. 3). In this region, expression of Kir2.1 slightly increased the percentage of cells in stationary phase (Fig. $6 F$ ). Finally, we analyzed GC-P in the deep GCL. Here, Kir2.1 expression had again no detectable effect on straightness, displacement and stationary phases but a small but significant increase in the speed of nuclear translocations (Fig. 6G). As an

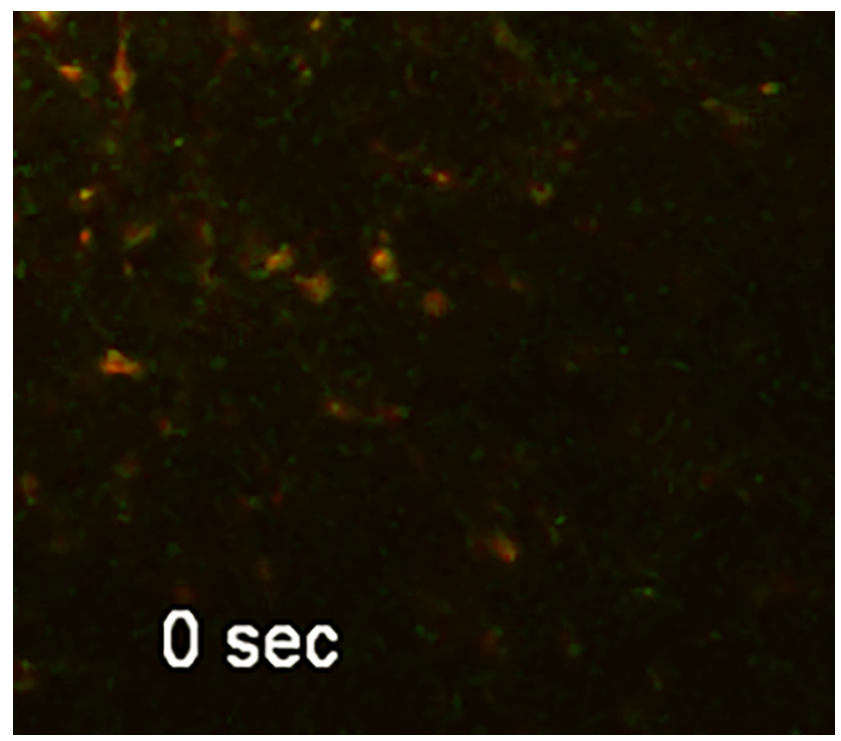

Movie 9. Example of a 2-min recording of calcium activity performed at the exit of the RMS-OB $7 \mathrm{~d}$ after medial electroporation. GCaMP6s fluorescence is in green, tdTomato in red. [View online]

additional control, we compared the migration of cells electroporated with the Kir mutant plasmid (in CD1 mice) and cells electroporated with a Cre-RNA in Ai14 (C57bl6). We observed small differences, with a slight increase of the mean displacement $\left(16.9 \pm 8.9\right.$ vs $21.5 \pm 10.6 ;$ LMEM, $\left.\chi^{2}(1)=4.2, p=0.04\right)$, and of the percentage of time spent in stationary phases $(73.2 \pm 17.2 \mathrm{vs}$ $60.6 \pm 19.5$; LMEM, $\left.\chi^{2}(1)=6.6, p=0.01\right)$ nevertheless the speed of nuclear translocation was unchanged $(43.9 \pm 13.3$ vs $42.5 \pm$ 10.7; LMEM, $\left.\chi^{2}(1)=0.9, p=0.3\right)$. While this may indicate an effect of the Kir2.1 mutant form, the absence of effect of nifedipine incubation and the relative absence of effect of the Kir2.1 channel compared with the mutant form, overall suggest that blocking neuronal activity has very little effect on the migration of GC-P.

We then tested whether neuronal activity controls the recruitment of GC-P and PGN-P in the OB layers. To test this hypothesis, we overexpressed Kir2.1 in GC-P and PGN-P by lateral or medial electroporation and analyzed neuronal distribution in the $\mathrm{OB}$ at $8 \mathrm{dpe}$, when migratory precursors leave the RMS-OB and settle in the $\mathrm{OB}$ (Fig. $7 A, B$ ). Importantly, and as shown in our previous experiments (Fig. $4 F, G$ ), Kir2.1 overexpression significantly decreases intrinsic calcium activity in both populations. We also performed experiments at 12 dpe when more cells had reached the $\mathrm{OB}$, to test for a potential cumulative effect.

At 8 dpe, the number of Kir2.1-expressing cells was significantly higher in the RMS-OB whereas less cells were present in the GCL (Fig. 7C). This effect was specific to GC-P, as distribution was unaffected in PGN-P after medial electroporation (Fig. 7D). At 12 dpe, when more cells entered the OB, this altered distribution in the GC population was slightly more pronounced (Fig. 7C) and, again, not detectable in PGN-P (Fig. 7D). At both time points, cell distribution in the GL was unaffected (Fig. 7C,D).

Finally, we asked whether blocking activity and/or the subsequent misdistribution of GCs had an impact on cell survival. To this end we overexpressed Kir2.1 and analyzed the expression of cleaved caspase 3 (c-caspase 3), a marker of apoptosis at 8 and 12 dpe in the RMS, the GCL and the GL (Fig. $8 A, B$ ). In agreement with previous studies, we found that the overall level of $c$-caspase 
A

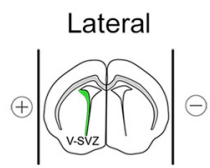

B

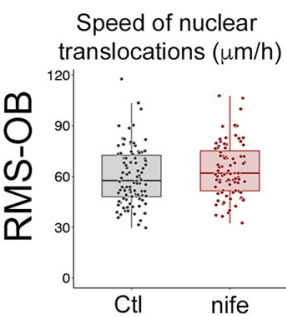

C

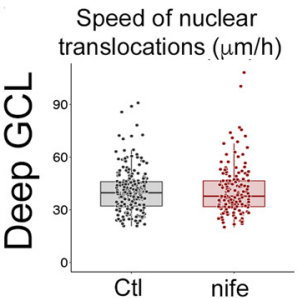

D

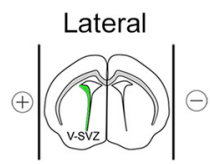

E

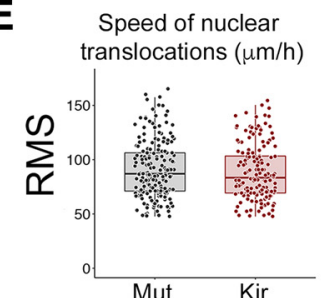

F

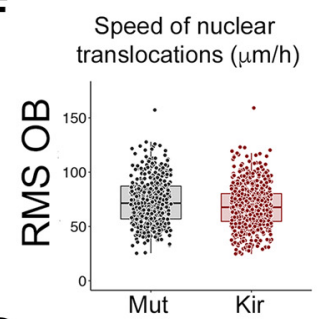

G
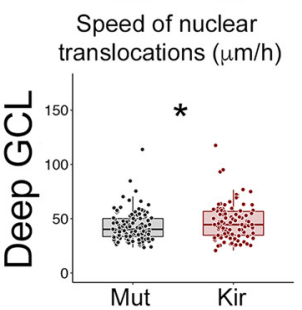

\section{$\begin{aligned} \text { P0 } & 7-8 \text { dpe } \\ \text { Lateral } & \text { Sacrifice: }\end{aligned}$ \\ Elpo Cre in migration recording \\ Rosa-tdTomato with Nifedipine}
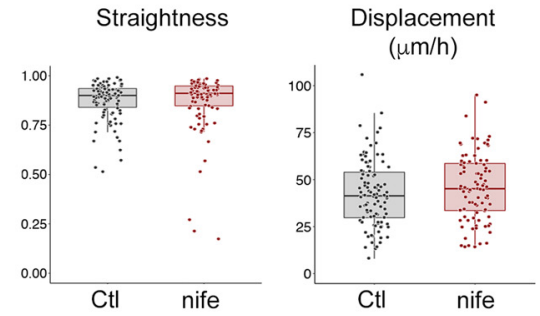

Stationary phases

(\%)
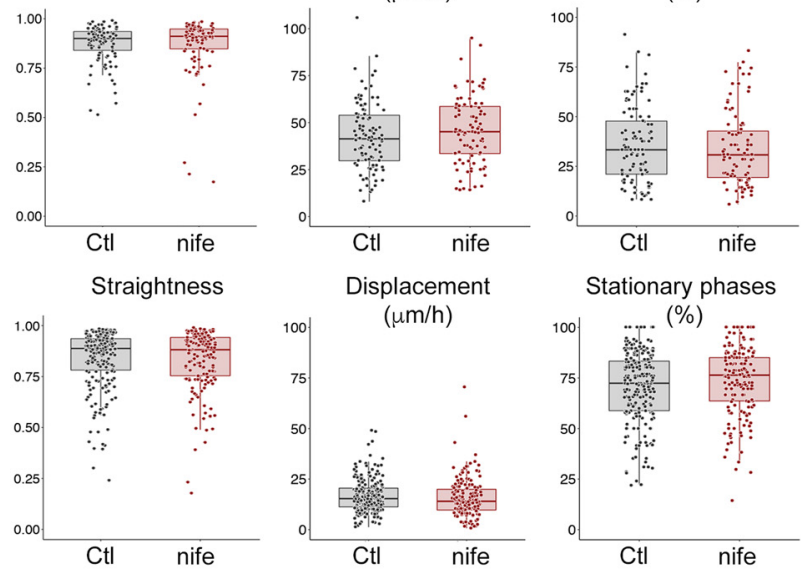

Displacement $(\mu \mathrm{m} / \mathrm{h})$
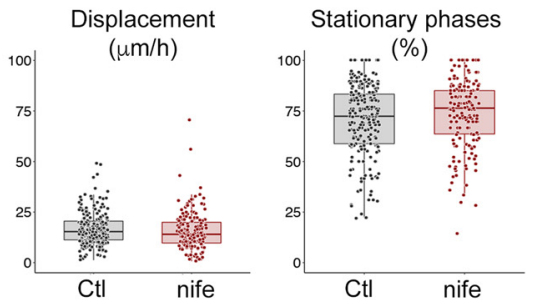

\section{$\stackrel{\text { P0 }}{\stackrel{\text { 7-8 dpe }}{\stackrel{1}{\text { Lateral }}} \text { Sacrifice: }}$ \\ Elpo Kir2.1/ migration recording \\ Kir mutant}
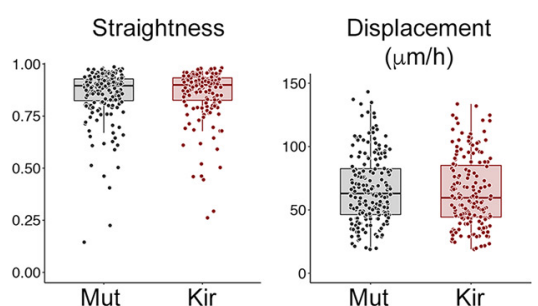

Stationary phases

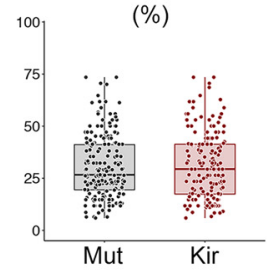

Straightness

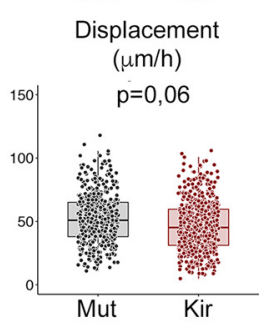

Stationary phases
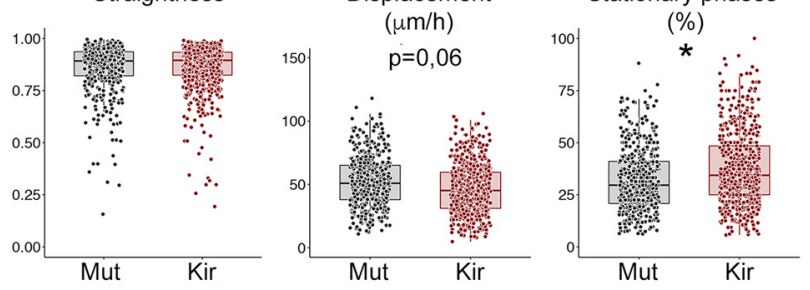

Displacement
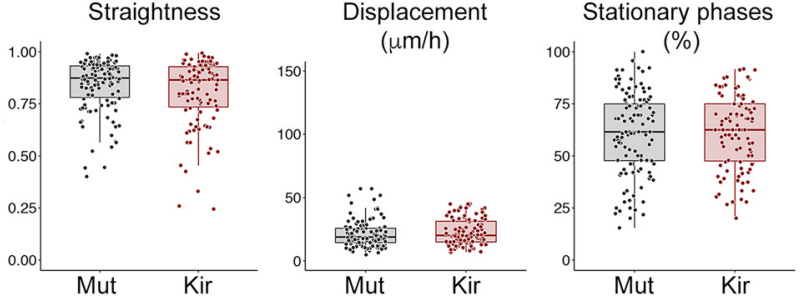

Figure 6. Effect of decreased neuronal activity on GC-P migration A, PO Rosa-tdTomato animals received lateral electroporation with Cre recombinase to label newborn cells. Mice were then killed at 7-8 dpe and the parameters of migration of GC-P were assessed on acute brain slices in the RMS-OB and deep GCL, with or without nifedipine (10 $\mu \mathrm{m})$. $\boldsymbol{B}$, Quantification of migration parameters in the RMS-OB. $N=102 / 91$ cells on four animals. Speed of nuclear translocation $\left[60.15 \pm 16.7\right.$ vs $64 \pm 16 ;$ LMEM, $\chi^{2}(1)=0.6, p=0.44$ (group effect)], straightness $\left[0.87 \pm 0.10\right.$ vs $0.87 \pm 0.15 ; \mathrm{LMEM}, \chi^{2}(1)=0.3, p=0.61$ (group effect)], mean displacement [42.4 \pm 17.6 vs $46.1 \pm 17.7 ; \mathrm{LMEM}, \chi^{2}(1)=0.4, p=0.54$ (group effect)], or percentage of time spent in stationary phases [35.9 \pm 19.1 vs $33.9 \pm 18.9$; LMEM, $\chi^{2}(1)=0.1, p=0.75$ (group effect)] were similar between both conditions. C, Quantification of migration parameters in the deep GCL. $N=225 / 172$ cells on four animals. Speed of nuclear translocation [40.8 \pm 12.4 vs $40.8 \pm 13.7 ;$ LMEM, $\chi^{2}(1)=0.05, p=0.83$ (group effect)], straightness [0.84 \pm 0.14 vs $0.83 \pm 0.15$; LMEM, $\chi^{2}(1)=0.07, p=0.79$ (group effect)], mean displacement [16.6 \pm 8.1 vs $15.7 \pm 9.4$; LMEM, $\chi^{2}(1)=0.4, p=0.52$ (group effect)], or percentage of time spent in stationary phases $\left[70.8 \pm 17.5\right.$ vs $73.8 \pm 16.8$; LMEM, $\chi^{2}(1)=0.81, p=0.37$ (group effect)] were similar between both conditions. $D, P 0$ CD1 animals received lateral electroporation with either a Kir2.1 mutant or a functional Kir2.1 encoding plasmid. Mice were then killed at 7-8 dpe, and the parameters of migration of GC-P were assessed on acute brain slices in the RMS, RMS-OB, and deep GCL. $\boldsymbol{E}$, Quantification of migration parameters in the RMS. $N=195 / 162$ cells on four animals. Speed of nuclear translocation $\left[91.5 \pm 26.7 \mathrm{vs} 88.5 \pm 25.6 ; \mathrm{LMEM}, \chi^{2}(1)=0.05\right.$, $p=0.8142$ (group effect)], straightness $\left[0.86 \pm 0.124\right.$ vs $0.86 \pm 0.122 ;$ LMEM, $\chi^{2}(1)=0.005, p=0.94$ (group effect)], mean displacement $[67.2 \pm 27.8$ vs $64.6 \pm 27.5 ;$ LMEM, 
A

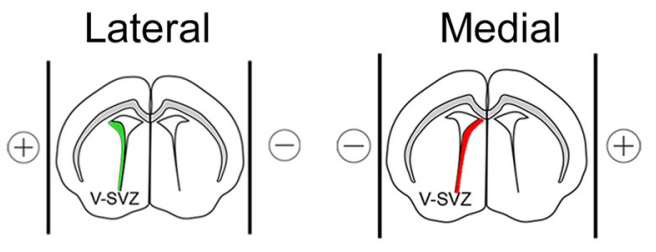

C

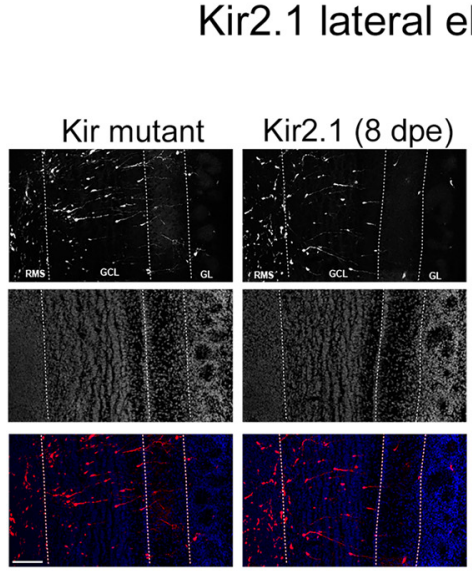

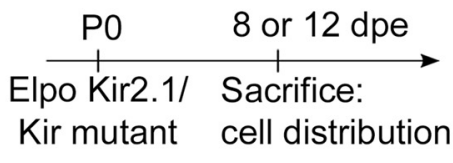

B

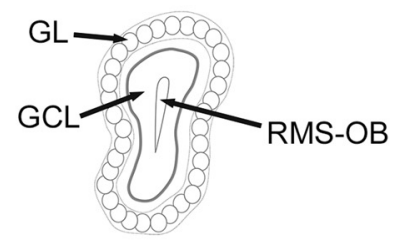

D

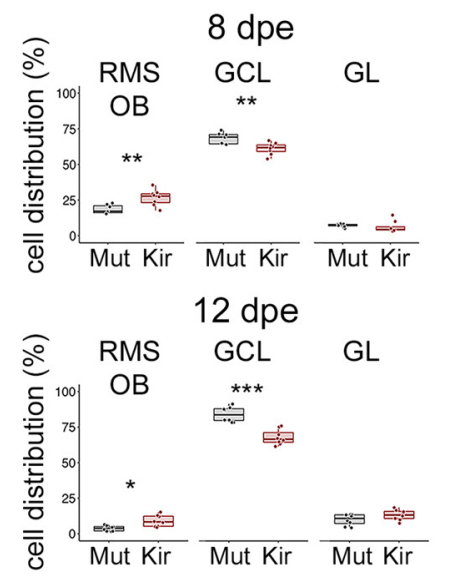

Kir2.1 medial electroporation

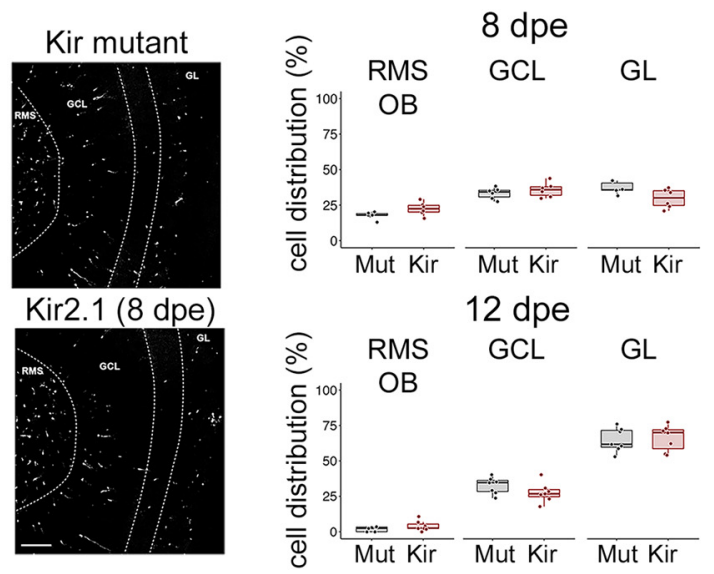

Figure 7. Effect of Kir2.1 expression on cellular distribution. A, PO CD1 animals received lateral or medial electroporation with either a Kir2.1 mutant or a functional Kir2.1 encoding plasmid. Mice were then killed either at 8 or 12 dpe. and the cellular distribution of tdTomato cells was assessed. B, Cellular distribution was observed on fixed coronal brain slices and cells attributed to the RMS, GCL, or GL. C, left, OB coronal sections of Kir2.1 mutant (Mut) and Kir2.1 (Kir) laterally electroporated animals at 8 dpe. Dotted lines separate OB layers. DAPI stains cell nuclei. Scale bar: $100 \mu \mathrm{m}$. Right, Quantification of the cellular distribution at $8 \mathrm{dpe}(N=7$ animals) and $12 \mathrm{dpe}(N=8$ animals) in each OB layer showing an increased percentage of Kir2.1 cells in the RMS ( 8 dpe: $18.5 \pm 3.1$ vs $26.7 \pm 5.5$, Mann-Whitney-Wilcoxon test, $p=0.006,12$ dpe: $9 \pm 4.1$ vs $3.8 \pm 1.9$, Mann-Whitney-Wilcoxon test, $p=0.007$ ) and a decrease in the GCL ( 8 dpe: $68.2 \pm 4$ vs $61.2 \pm 4.2$, Mann-Whitney-Wilcoxon test, $p=0.009 ; 12$ dpe: $84 \pm 5.2$ vs $68 \pm 5.3$, Mann-Whitney-Wilcoxon test, $p=0.00015$ ). Cellular distribution in the GL were similar for both conditions at 8 dpe (7.2 \pm 1.2 vs $6.13 \pm 4$, Mann-Whitney-Wilcoxon test, $p=0.12)$ and 12 dpe $(9.9 \pm 3.9$ vs $13.2 \pm 3.7$, Mann-Whitney-Wilcoxon test, $p=0.13)$. $D$, left, 0 B coronal sections of Kir2.1 mutant and Kir2.1 medially electroporated animals at 8 dpe. Dotted lines separate OB layers. Scale bar: $100 \mu \mathrm{m}$. Right, Quantification of the cellular distribution at 8 dpe ( $N=6$ animals) and $12 \mathrm{dpe}(N=7$ animals) in each $O B$ layer. No significant effect on cellular distribution was observed in this condition, neither in the RMS ( 8 dpe: $17.8 \pm 2.5$ vs $22.5 \pm 4.7$, Mann-Whitney-Wilcoxon test, $p=0.07,12$ dpe: $1.9 \pm 1.8$ vs $4.1 \pm 3.6$, Mann-Whitney-Wilcoxon test, $p=0.33$ ), GCL (8 dpe: $33.3 \pm 4$ vs $35.87 \pm 5.5$, Mann-Whitney-Wilcoxon test, $p=0.48,12$ dpe: $32.6 \pm 5.9$ vs $27.7 \pm 7$, Mann-Whitney-Wilcoxon test, $p=0.16$ nor GL (8 dpe: $37.2 \pm 4.2$ vs $29.7 \pm 6.7$, Mann-Whitney-Wilcoxon test, $p=0.132,12$ dpe: $64.8 \pm 8.4$ vs $3.8 \pm 1.9$, Mann-Whitney-Wilcoxon test, $p=0.71)$.

3 expressing cells was very low (Petreanu and Alvarez-Buylla, 2002; Yamaguchi and Mori, 2005; Platel et al., 2010). However, $8 \mathrm{~d}$ after lateral electroporation, we detected a slight but significant increase of c-caspase 3 immunoreactive cells among GC-Pexpressing Kir2.1 in the GCL. At 12 dpe, this effect was even more pronounced in the GCL (Fig. $8 C$ ) and we observed a strong but not significant tendency in the RMS-OB. Importantly, we

\section{$\leftarrow$}

$\chi^{2}(1)=0.03, p=0.86$ (group effect)], or percentage of time spent in stationary phases $\left[30.5 \pm 14.9\right.$ vs $31.3 \pm 16 ;$ LMEM, $\chi^{2}(1)=0.013, p=0.91$ (group effect)] were similar between both populations. $\boldsymbol{F}$, Quantification of migration parameters in the RMS-OB. $N=472 / 602$ cells on 13 animals. Speed of nuclear translocation [73 \pm 20.7 vs $68.2 \pm 19.8$; LMEM, $\chi^{2}(1)=2.5, p=0.1138$ (group effect)], straightness [0.86 \pm 0.11 vs $0.862 \pm 0.12$; LMEM, $\chi^{2}(1)=0.08, p=0.78$ (group effect)], or mean displacement $[52.5 \pm 19.8$ vs $46.4 \pm 19.5$; LMEM, $\chi^{2}(1)=3.5, p=0.061$ (group effect)] were not significantly different between the two groups. However, GC precursors expressing Kir2.1 in the RMS-OB slightly increased their time spent in stationary phases $[32.2 \pm 15.2$ vs $37.7 \pm 17.6$; LMEM, $\chi^{2}(1)=4.1, p=0.043$ (group effect)]. G, Quantification of migration parameters in the deep GCL. $N=119 / 103$ cells on 5 animals. Straightness [0.84 \pm 0.13 vs $0.80 \pm 0.16$; LMEM, $\chi^{2}(1)=1.9, p=0.17$ (group effect)], mean displacement $[21.5 \pm 10.6$ vs $22.8 \pm 10.4$; LMEM, $\chi^{2}(1)=0.02, p=0.88$ (group effect)], or percentage of time spent in stationary phases [60.6 \pm 19.5 vs $61.7 \pm 17.6$; LMEM, $\chi^{2}(1)=0.12, p=0.73$ (group effect)] were similar between both populations. A slight increase of the speed of nuclear translocation is observed in the deep GCL for KIR2.1-expressing cells [42.6 \pm 13.4 vs $46.9 \pm 16.3$, LMEM, $\chi^{2}(1)=4.7, p=0.031$ (group effect)]. did not observe any increase in apoptosis after expression of Kir2.1 in PGN-P at 8 or 12 dpe (Fig. $8 D$ ).

Overall, we conclude that activity in GC-P has very little effect on neuronal migration, but is important for their recruitment and survival in the GCL. PGN-P are not affected by the inhibition of activity. Taken together, these results suggest that activity in GC-P at the exit of the RMS is a controlling factor in the recruitment and positioning process in the $\mathrm{OB}$.

\section{Discussion}

Combining targeted electroporation and time-lapse two-photon imaging, we were able for the first time to compare and analyze migrating precursors of the two main $\mathrm{OB}$ interneuron subtypes. Our deep characterization of the tangential and radial migration of these two cell types did not show any obvious morphologic or kinetic parameter that allows to differentiate them, suggesting that GC-P and PGN-P share the same mode of migration to reach the OB. However, we identified a dramatic increase of calcium activity in GC-P at the time of switching between tangential to radial migration. This calcium activity was found to be mostly mediated by a voltage-dependent extracellular calcium entry through L-type calcium channels, meaning that precursors were depolarized. Blocking this activity by dampening neuronal excitability with nifedipine or by overexpressing Kir channels did not strongly alter GC-P migration. Interestingly, blocking activity led 
A

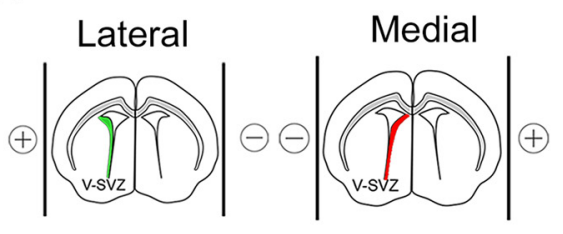

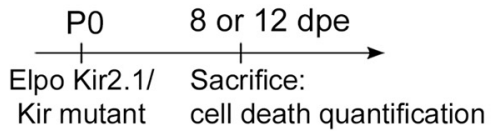

$\begin{array}{ll}\text { Kir mutant } & \text { cell death quantification }\end{array}$
B

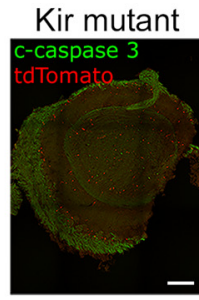

Kir2.1

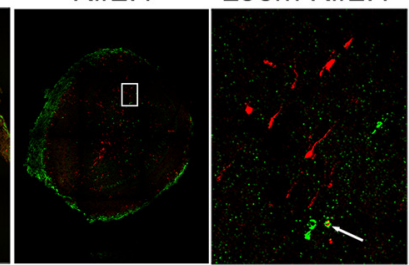

C

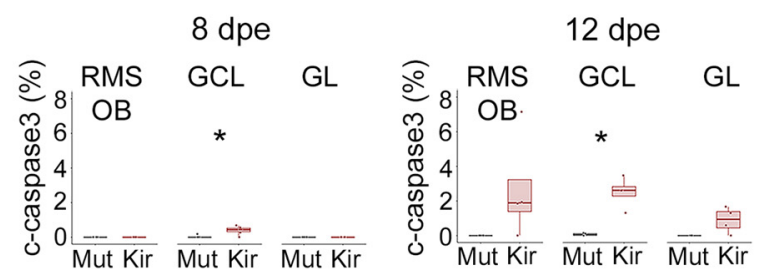

Kir2.1 lateral electroporation
D

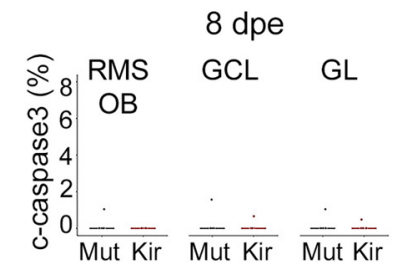

Kir2.1 medial electroporation

8 dpe 12 dpe

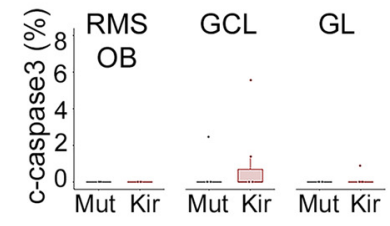

Figure 8. Effect of Kir2.1 expression on neuronal cell death. $\boldsymbol{A}$, PO CD1 animals received lateral or medial electroporation with either a Kir2.1 mutant or a functional Kir2.1 encoding plasmid. Mice were then killed at 8 or 12 dpe and the level of apoptotic cell death in tdTomato cells was quantified. $\boldsymbol{B}, 0$ B coronal sections of Kir mutant and Kir2.1 labeled with c-caspase 3 at 12 dpe. Note the presence of a double-labeled cell in the Kir condition. $\boldsymbol{C}, \boldsymbol{D}$, Quantification of the percentage of c-caspase 3-positive cells among lateral (C) or medial ( $\boldsymbol{D}$ ) electroporation of Kir2.1 (Kir) or Kir2.1 mutant (Mut) cells per $0 B$ region at 8 dpe (lateral: $N=6$ animals; medial: $N=6$ animals) or 12 dpe (lateral: $N=7$ animals; medial: $N=4$ animals). There is an increase of c-caspase 3 -positive cells after lateral electroporation of Kir2.1 in the GCL at 8 dpe $(0.03 \pm 0.07$ vs $0.4 \pm 0.24$, Mann-Whitney-Wilcoxon test, $p=0.017)$ and 12 dpe $(0.07 \pm 0.08$ vs $2.5 \pm 0.9$, MannWhitney-Wilcoxon test, $p=0.029$ ) but not after medial electroporation.

to a progressive accumulation of GC-P in the RMS OB at the expanse of the GCL and to a increase in cell death in radially migrating GC-P. In contrast, blocking neuronal activity in PGN precursors did not affect their cellular distribution or cell death. Overall, these experiments suggest that neuronal activity in GC precursors is necessary for their recruitment in the $\mathrm{OB}$, while this is not the case for PGN-P.

It was shown in the $\mathrm{OB}$ that olfactory training (Mouret et al., 2008) or overexpression of $\mathrm{NaChBac}$ (Lin et al., 2010) could increase recruitment during synaptic integration. In sharp contrast, it was recently demonstrated that in physiological condition, selection during synaptic integration is nearly absent (Platel et al., 2019) pointing to a first selection step occurring before integration (Biebl et al., 2000; Platel et al., 2010). In agreement with these latter studies, our results suggest that survival of neuroblasts during radial migration is dependent on neuronal activity. Importantly, this phenomenon seems to be subpopulation specific. While it is known that layer positioning of different cortical interneurons depends on neuronal activity (De Marco García et al., 2011; Lodato et al., 2011), it is unclear to which extent this is a result of altered recruitment or differential cell death.

Our study also show a subtype-specific effect of neuronal activity during the switch between tangential to radial migration. It should be noted that the expression of the calcium indicator (GCaMP6s) could have disturbed $\mathrm{Ca}^{2+}$ currents in our subpopulations, and in particular L-type VGCCs mediated calcium currents (Bellion et al., 2005; Steinmetz et al., 2017; Yang et al., 2018). However, we believe that the simplest hypothesis is that this would affect our two subpopulations similarly, therefore not impacting our conclusions. Also, we found that the expression of the mutant form of Kir2.1 had a mild effect on migration compared with other experiments using Cre electroporation in Rosa tdTomato mice. This suggests that overexpressing even a non functional channel could affect the cells. It is thus important to compare the effect of the normal Kir2.1 to the mutant form, to make sure that the effects are because of increased excitability rather than because of the overexpression itself. It should also be noted that the comparison between the mutant and the wildtype forms was performed in two independent experiments and in in different mouse lines, making the comparison more difficult.

Despite our efforts, we could not identify a specific signal explaining the large increase in neuronal activity at the RMS exit for the GC-P. It was found for migrating glutamatergic cortical neurons that transient synaptic connections occur during migration and that these connections were impacting migration (Ohtaka-Maruyama et al., 2018). In our pharmacological screen, neither glutamate nor GABA nor glycine receptors' antagonists were able to block the large rise in calcium activity. A tempting hypothesis would be that rather than being dependent on extracellular signals (such as neurotransmitter), this activity could be the consequence of neuroblast maturation. Interestingly, our observations suggest that cells can migrate for a significant time (at least $8 \mathrm{~h}$ ) in the RMS of the OB before exiting in the GCL. In this hypothesis, migrating cells in the RMS would need to reach a certain threshold of maturation, allowing proper intrinsic activity, which would in turn favor their switch to radial migration.

Contrary to GC precursors, PGN precursors were not affected by Kir2.1 mediated hyperpolarization suggesting that the recruitment of future PGNs is not activity dependent. This is in line with findings showing that knocking out serotonin receptors did no affect cell density in the GL (García-González et al., 2017) and results showing that the migration of PGN precursors in the GL does not depend on sensory activity (Liang et al., 2016). Of note, this later study also showed that PGN neurons migrate for an extended period in the GL while most GCs had settled. This is coherent with the hypothesis that the maturation of GCs and PGNs during migration follows a different time course.

To our knowledge, there is no candidate factor known to regulate the addressing of PGN to their specific layer. New advances in single cell transcriptomics coupled to our specific targeting of the two subpopulations might allow to decipher which molecular pathways differ between PGN and GC precursors during their radial migration.

In conclusion, we show that intrinsic neuronal activity early during neuronal development, when neurons are still migrating, can control the recruitment of specific interneuron subtypes to their target layer. 


\section{References}

Bellion A, Baudoin JP, Alvarez C, Bornens M, Métin C (2005) Nucleokinesis in tangentially migrating neurons comprises two alternating phases: forward migration of the Golgi/centrosome associated with centrosome splitting and myosin contraction at the rear. J Neurosci 25:5691-5699.

Biebl M, Cooper CM, Winkler J, Kuhn HG (2000) Analysis of neurogenesis and programmed cell death reveals a self-renewing capacity in the adult rat brain. Neurosci Lett 291:17-20.

Boutin C, Diestel S, Desoeuvre A, Tiveron MC, Cremer H (2008) Efficient in vivo electroporation of the postnatal rodent forebrain. PLoS One 3:e1883.

Bugeon S, de Chevigny A, Boutin C, Coré N, Wild S, Bosio A, Cremer H, Beclin C (2017) Direct and efficient transfection of mouse neural stem cells and mature neurons by in vivo mRNA electroporation. Development 144:3968-3977.

De Marco García NV, Karayannis T, Fishell G (2011) Neuronal activity is required for the development of specific cortical interneuron subtypes. Nature 472:351-355.

Fernández ME, Croce S, Boutin C, Cremer H, Raineteau O (2011) Targeted electroporation of defined lateral ventricular walls: a novel and rapid method to study fate specification during postnatal forebrain neurogenesis. Neural Dev 6:13.

García-González D, Khodosevich K, Watanabe Y, Rollenhagen A, Lübke JHR, Monyer H (2017) Serotonergic projections govern postnatal neuroblast migration. Neuron 94:534-549.e9.

Inada $H$, Watanabe $M$, Uchida $T$, Ishibashi $H$, Wake $H$, Nemoto $T$, Yanagawa Y, Fukuda A, Nabekura J (2011) GABA regulates the multidirectional tangential migration of GABAergic interneurons in living neonatal mice. PLoS One 6:e27048.

Liang Y, Li K, Riecken K, Maslyukov A, Gomez-Nicola D, Kovalchuk Y, Fehse B, Garaschuk O (2016) Long-term in vivo single-cell tracking reveals the switch of migration patterns in adult-born juxtaglomerular cells of the mouse olfactory bulb. Cell Res 26:805-821.

Lin CW, Sim S, Ainsworth A, Okada M, Kelsch W, Lois C (2010) Genetically increased cell-intrinsic excitability enhances neuronal integration into adult brain circuits. Neuron 65:32-39.

Lodato S, Rouaux C, Quast KB, Jantrachotechatchawan C, Studer M, Hensch TK, Arlotta P (2011) Excitatory projection neuron subtypes control the distribution of local inhibitory interneurons in the cerebral cortex. Neuron 69:763-779.

Lois C, Alvarez-Buylla A (1994) Long-distance neuronal migration in the adult mammalian brain. Science 264:1145-1148.

Merkle FT, Mirzadeh Z, Alvarez-Buylla A (2007) Mosaic organization of neural stem cells in the adult brain. Science 317:381-384.
Morin X, Jaouen F, Durbec P (2007) Control of planar divisions by the Gprotein regulator LGN maintains progenitors in the chick neuroepithelium. Nat Neurosci 10:1440-1448.

Mouret A, Gheusi G, Gabellec MM, de Chaumont F, Olivo-Marin JC, Lledo PM (2008) Learning and survival of newly generated neurons: when time matters. J Neurosci 28:11511-11516.

Nam SC, Kim Y, Dryanovski D, Walker A, Goings G, Woolfrey K, Kang SS, Chu C, Chenn A, Erdelyi F, Szabo G, Hockberger P, Szele FG (2007) Dynamic features of postnatal subventricular zone cell motility: a twophoton time-lapse study. J Comp Neurol 505:190-208.

Ohtaka-Maruyama C, Okamoto M, Endo K, Oshima M, Kaneko N, Yura K, Okado H, Miyata T, Maeda N (2018) Synaptic transmission from subplate neurons controls radial migration of neocortical neurons. Science 360:313-317.

Petreanu L, Alvarez-Buylla A (2002) Maturation and death of adult-born olfactory bulb granule neurons: role of olfaction. J Neurosci 22:6106-6113.

Platel JC, Dave KA, Gordon V, Lacar B, Rubio ME, Bordey A (2010) NMDA receptors activated by subventricular zone astrocytic glutamate are critical for neuroblast survival prior to entering a synaptic network. Neuron 65:859-872.

Platel JC, Angelova A, Bugeon S, Wallace J, Ganay T, Chudotvorova I, Deloulme JC, Béclin C, Tiveron MC, Coré N, Murthy VN, Cremer H (2019) Neuronal integration in the adult mouse olfactory bulb is a nonselective addition process. Elife 8:e44830.

Saghatelyan A, Roux P, Migliore M, Rochefort C, Desmaisons D, Charneau P, Shepherd GM, Lledo PM (2005) Activity-dependent adjustments of the inhibitory network in the olfactory bulb following early postnatal deprivation. Neuron 46:103-116.

Steinmetz NA, Buetfering C, Lecoq J, Lee CR, Peters AJ, Jacobs EAK, Coen P, Ollerenshaw DR, Valley MT, de Vries SEJ, Garrett M, Zhuang J, Groblewski PA, Manavi S, Miles J, White C, Lee E, Griffin F, Larkin JD, Roll K, et al. (2017) Aberrant cortical activity in multiple GCaMP6expressing transgenic mouse lines. eNeuro 4:ENEURO.0207-17.2017.

Wong FK, Bercsenyi K, Sreenivasan V, Portalés A, Fernández-Otero M, Marín O (2018) Pyramidal cell regulation of interneuron survival sculpts cortical networks. Nature 557:668-673.

Xue M, Atallah BV, Scanziani M (2014) Equalizing excitation-inhibition ratios across visual cortical neurons. Nature 511:596-600.

Yamaguchi M, Mori K (2005) Critical period for sensory experience-dependent survival of newly generated granule cells in the adult mouse olfactory bulb. Proc Natl Acad Sci USA 102:9697-9702.

Yang Y, Liu N, He Y, Liu Y, Ge L, Zou L, Song S, Xiong W, Liu X (2018) Improved calcium sensor GCaMP-X overcomes the calcium channel perturbations induced by the calmodulin in GCaMP. Nat Commun 9:1504. 